\title{
HIERARCHICAL THINKING - A COGNITIVE TOOL FOR GUIDING COHERENT DECISION MAKING IN DESIGN PROBLEM SOLVING
}

\author{
Grietjie Haupt ${ }^{1, *}$ \\ ${ }^{1}$ Department of Science, Mathematics and Technology EducationUniversity of PretoriaHillcrestSouth Africa \\ *grietjie.haupt@up.ac.za
}

\begin{abstract}
This paper builds on two concept, the first of which is the extended information processing model of expert design cognition. This proposes twelve internal psychological characteristics interacting with the external world of expert designers during the early phases of the design process. Here, I explore one of the characteristics, hierarchical abstraction, and adapt it into an alternative ontological model of decision making. The model serves as an in-depth descriptor of how designers from different domains transform their mental states using judgment and decision making through hierarchical abstraction. The second concept entails an expansion of the idea of synergistic vertical transformation as a framework for mapping expert designers' design process. Here, I focus on hierarchical decision making as multi-directional, and inter-relating the internal and external world of designers. In doing so, I provide a coding tool for researchers interested in exploring designers' complex decision making processes. Concurrently, the model serves as decision making tool in design and technology education classrooms. As such, the paper focuses on the ontology of conceptual structures that support the early phases of the design process. This was based on empirical research.
\end{abstract}

Keywords: Design cognition, Decision making, Hierarchical thinking, 4-Level decision making tool, Problem solving, Intentions, Multi-directional transformation

\section{Introduction}

Designing is an act that involves dynamic processes and sub-processes in which adaptive and flexible thinking is required. One of the primary goals of research and learning processes in such dynamic contexts is to attain optimal effectiveness from the generation, conception, transfer and implementation of new knowledge. This effectiveness can be determined by the designer's quality and speed of connection making between the context-bound intention(s) of an artefact or system, the choice of appropriate physical elements defining the artefacts' unique form and structure, judgment of expected effective behaviour under particular conditions, and the practicalities involved in the implementation or construction of the artefact or system. Wide evidence exists that the decision making tools used by engineers (Eder, 2012), business management systems (Fox, Cooper, \& Glasspool, 2013) and knowledge managers (Gavrilova, Leshcheva, \& Strakhovich, 2015) used to address the focus of this paper are positively connected with the quality and speed of connection making and decision making. Decision making tools serve as a visual cognitive instrument that simplifies complex connection making processes both in educator/learner interaction, designer/client interaction and within research communities. These assist in the management of the knowledge used during the decision making process. Particular interest in such decision making tools that codify knowledge can be observed in engineering, industrial design, architecture and business management practice, and education 
where continuous feedback from within individual and group relationships is provided. However, many traditional tools represent a static approach to the design process in that they encode knowledge and processes in an a priori manner. This denies the dynamics involved in transforming tentative choices into committed decisions resulting from an extended world that encompasses individual designing by processing of internal and external sources of knowledge. Part of their knowledge application during their design processes entails that designers consider multiple dimensions of artefacts, including suitable combinations of artefacts' multi-disciplinary philosophical aspects, functional-behavioural intentions, form or physical aspects, and the practical implementation potential of the combinations decided on.

There is a developing understanding of how expert designers maintain internal consistency amongst the multiple themes around which their thoughts revolve when designing artefacts (Fox et al., 2013; Hastie, 2001). Likewise, there is an emerging understanding of what drives their judgements, choices and decisions to ensure coherence and how their thoughts are structured, as found in management literature (Tversky \& Kahneman, 1974, 1986; Tversky \& Simonson, 1993). As a result, design researchers, educationalists and practitioners construct thinking tools to enhance designers' and students' knowledge application and resultant design methodology. As decision making involves the consideration of many alternatives, and making trade-offs, designing becomes knowledge and labour intensive, and these tools are believed to alleviate the cognitive load of designers. They are furthermore believed to help save time, keep designers focused, and consequently help them to make better decisions (Fox, et al., 2013, p.2). With this approach in mind, I intended to show how an ontological framework for decision making, resulting in a novel multi-directional 4-level hierarchical decision making model, can serve as an alternative thinking and teaching tool across design domains and levels of expertise.

The aim of this paper was therefore threefold: firstly, it was intended to clarify some issues around the limitations in current decision making tools reported on in the literature from a design cognition perspective. Secondly, it aimed to create an awareness in design educators of the cognitive patterns in the decision making process as prompted by internal and external information entering students' information processing system. In turn, this might thirdly provide design educators with a practical visual decision making tool to be administered manually.

This could help educators to foster a self-awareness amongst students of the close relationship between their knowledge, the hierarchical structure of their thoughts, and its correlation with the transformation of their mental states during the early phases of the design process based on philosophical reasoning. As such, it addresses some of the gaps in existing design related frameworks that are limiting due to their bias towards epistemology. Some frameworks are limited by their reductionist emphasis on utility. Visual ontological tools, including mind maps and concept maps, focus on the structured classification of ontological components without accounting for the complexity and individuality of the psychological processes of decision making.

In an attempt to fill this gap, I used Goel's (1995) general psychological theory of abstraction hierarchies by connecting abstraction with intentions. I also expand on an earlier (2013) conceptualisation of the synergetic vertical transformation space of expert engineering design processes. I adopted an extended cognition approach, which resonates with Gero and 
Kannengieser's (Gero \& Kannengieser, 2004) situated approach to framing the design process. In so doing, I provide an ontological model that overarches the internal and external sources of knowledge and internal and external cognitive processes. Therefore, the philosophical concept 'ontology', which aims to study the nature of existing entities - physical or abstract (Hofweber, 2014), here serves as a focal point. Ontologies can be used to describe the nature of any particular design thinking structure. However, it is not possible to consider such ontologies without also acknowledging the importance of design and technological knowledge (epistemology), design methods (methodology) and designers'/clients' free will (volition) in their decision making process. The argument in this paper is that, in order to support researchers, practitioners, educators and students in accounting for the efficient structuring of thoughts, we need a well-balanced decision making tool that includes elements of all four philosophical focal areas.

In light of the focus of this paper, namely, to explore the cognitive mechanisms involved in acts of hierarchical judgment and decision making, the proposed tool assists in accounting for:

- The centrality of intentions as anchoring and adjustment mechanism;

- The significance of representations;

- The multi-directional transformation of thoughts;

- The influence of internal and external sources of knowledge;

- Dynamic processes and adaptive thinking; and

- Generic processes across domains and expertise levels.

This paper is not limited in support of a particular design methodology. Instead, the inquiry here is focused rather on the success and failure of the role of a framework in mapping multidirectional hierarchical thinking, as well as its potential use as an instructional tool in design education contexts. The model is aimed at solving problems of information overload, misunderstanding and incoherence in the early phases of the design process.

The purpose of this paper is therefore to propose an alternative decision making tool that is modelled on the four levels of abstraction found in the content of designers' thoughts during the early phases of the design process. This tool has developed from the empirical research conducted on expert designers from various disciplines, applying an extended cognition viewpoint. The study shows how the epistemological scope of designers' decision making processes encompass four levels of thinking, which can be accessed in their verbal and visual representations, and serves as content, as well as a multi-directional structuring instrument of their thought processes.

As such, I offer an extended cognition view of the generic psychological mechanisms involved in adapted decision-making with the purpose of answering the following questions:

1. What are the driving forces of decision making in the early phases of the design process?

2. What cognitive role does a multi-directional transformation process play in ensuring coherence?

3. How might we use an alternative ontological model of hierarchical decision making to support students' selection of information, relevance and proximity to intentions during the design process? 
In answering these questions, the paper is structured in the following manner: I first explore the limitations in current design literature focusing on decision making tools. Second, I discuss and present the theoretical implications of the 4-level decision making model as an ontological tool, supported by evidence from empirical research. The model is deconstructed by discussing its underlying assumptions, and structural and operational characteristics. Third, I present the model's potential as decision making tool in novice design environments and argue its ability to transfer some expert decision making skills across domains and levels of expertise.

\section{Decision making tools and their theoretical foundations}

Research that has explored the thinking tools that are currently emerging in the design and technology fields apply an ontological or epistemological approach, or a combination thereof. 'Ontology' here signifies 'the study of the most general features of what there is, and how the things there are relate to each other ...' (Hofweber, 2014). Here, I will consider the general features of an ontological framework for hierarchical decision making, and its lateral and vertical relationships with decision making processes in the early phases of the design process. As such, the framework serves as a foundational framework for the field of design, which means that it overlaps with the process of attaining knowledge, also known as meta-knowledge. This overlap stems from the widely acknowledged assumption that designers constantly need to search, find and apply appropriate knowledge and information in order to structure and solve given design problems. This search and find process entails identifying relevant knowledge, judging its relevance through comparison, evaluating and reversing transformations, and committing to decisions that proximate given intentions. 'Finding relevance' here resonates with the psychological notion of 'anchoring and adjustment heuristic' (Epley \& Gilovich, 2006). 'Anchoring' is defined as estimating a probability by starting from an initial reference point (for instance a particular intention) and then making adjustments in the direction that seems most appropriate. 'Adjustment' connects with the idea of intuitive 'judgment', which results in manipulation and development. However, in order to counter insufficient relevance, supporting the selection of the most useful information, researchers and design practitioners have for a long time been developing decision making tools to make the task of knowledge selection and decision making more efficient (Fox et al., 2013, p.3)

Historically, cognitive research involving judgment and decision making (Goldstein \& Hogarth, p.3-68) was known as utility theories. These were often used to conduct research and improve decision making practice in engineering (Eder, 2012; Katsikopoulos, 2009; Seram, 2013) and business management contexts (Fox et al., 2013). It was the accepted theory since the late 1940s, with many variations (Hastie, p.658). There is a lot of literature that discusses the inherent nature of such tools from different perspectives using varying classifications. These tools have been classified and include descriptive tools, which contain representations of the designed artefact's functional relationships. Behavioural models represent the response of individual components or their properties to a system in an initial disturbance. Deterministic models contain prescriptive and known functional relationships of physical components, while stochastic models are based on the probability of functional relationships. Imitative models, in the form of simulations, are used to analyse a specific problem. Finally, optimisation tools are prescriptive and are used to select the most appropriate approach to the choice of physical components. Conventional utility 
models are based on analytical and pairing methods of calculating the best or most suitable decisions made by designers. The foundation of these theories is a distinction between information about what designers want (their intentions), also known as 'utilities' or 'functional intentions', and what designers believe about how the selected concept, including the artefact's physical form, would proximate the expected behaviour of an artefact to solve a problem under particular conditions (Fox et al., 2013; Hastie, 2001). One example of such tools include the analytical hierarchical process (AHP) developed by Saaty (Kluge \& Malan, 2011, p.847-848) to help ordinary people make complex decisions. Saaty did this by providing alternatives to solve a particular problem and criteria for evaluation of the alternative. Pairwise comparisons of the elements of the hierarchy are conducted by the engineer to establish priorities amongst the elements. This is followed by a synthesis of the comparisons into a set of overall priorities for the hierarchy. Finally, consistency amongst the comparisons is tested and a decision is then made based on the results of the tests.

What all these utility type frameworks have in common is the rational expectancy principle. This provides for a static top-down selection process, and suggests that each potential choice should be holistically evaluated by weighting its expected satisfaction-dissatisfaction. These should be weighted with the probabilities that the consequences of each individual component included will occur and be experienced. Generally, according to Hastie (p.657), standards for the quality of such judgments are essentially based on accuracy of the correspondence between a judgment, and the criterion condition for achieving a goal or intention, which should be the aim of the judgment. Although these tools are different in their approach, what they furthermore share is their acknowledgement of multi-variants that are paired, as well as single or multiple levels that are vertically distributed in a top-down manner. However, they typically do not allow for the inclusion of lateral thinking and the generation of additional options.

They are all valuable to design practice and education in providing analytical tools to prioritise criterion, its sub-elements, and create a logic flow in the decision making process and provide concrete data. Although, Hastie (p.658) emphasises two limitations of the utility models depicting the ontology of the decision making processes. The first weakness is that they are incomplete. Many aspects of the decision process lie outside the framework's abstract, analytical comparison process, and do not account for the way in which the decision situation is understood or constructed by designers. Its abstract nature does not inform us about the courses of action that are under consideration in the choice set. We also do not know what the input information sources were at the outset, which physical elements were considered, which were rejected, and why. It also tends to say nothing about the trade-offs. The second weakness is that it does not provide a valid description of the microscopic detail of the decision making process. This means that it does not account for the reasoning process, the trade-offs and justifications involved in the judgements, evaluations, choices, and decisions made by the designer. In summary, the limitations of the majority of rational utility models is that they narrowly focus on mathematical equations of decision making and do not provide valid descriptions of the details of naturalistic human decision making processes that are necessary in fluid, uncertain and creative environments (Hastie, 2001, p.658), such as designing. Despite this limitation, utility theories still seem to be popular and in use in engineering education programmes, as well as in engineering practice (Katsikopoulos, 2009; Kluge \& Malan, 2011). 
The second type of ontological frameworks currently used by knowledge managers is that of visualisation of ontological structures, which is used in complex learning processes to transfer knowledge and understanding. These types of frameworks include mind mapping and concept mapping, which are typically used to support memorising and externalising the relationships between the various concepts contained in particular domains (Gavrilova et al., 2015, p.3883). The idea of using the visual structuring of ontologies, or any other information model, to improve the quality of knowledge transfer has gained recognition through cognitive and education researchers, including Buzan (2005), Sowa (1984), Jonassen (1998), and Conlan (2006). An example of the use of concept mapping in architectural design education is the WebPad program experimentally investigated by Oxman (2004). Its usefulness as a thinking map in a domain specific environment is based on the assumptions of constructivist learning. Instead of teaching students how to design, educators use concept mapping to teach them how and where to find appropriate knowledge. The ability of concept maps to organise and visually represent the requisite knowledge therefore allows designers/students to know where to find a particular type of knowledge, when to use it, and how to apply it when necessary. It is thus particularly useful to augment lectures and textual or visual study material in educational environments (Oxman, 2004, p.68) and to contribute to meta-knowledge or the knowledge of how to organise one's own knowledge. However, these types of tools do not offer the opportunity to define, trace and map the cognitive processes involved in the knowledge generation and connection making between the various content components of designers during the design process.

An example of an epistemological framework that frames designers' knowledge about the nature of artefacts is the 'dual nature of artefacts' framework, which originated in an initiative by Kroes and Meijers (2002). This framework is philosophical in nature and is not meant as a practical utility model. However, it resonates strongly with past conceptualisations of existing models that represent the function-behaviour-structure thinking system identified in 1960-1970s utility models. One of the reasons for these authors' philosophical interest seems to be a reaction to the inability of existing decision making models to provide logical arguments for the consideration of particular knowledge in design methodology. However, Kroes and Meijers (2002, p.3) acknowledge the following:

It is still a problem exactly how the intentional and the physical description of artifacts hang together. If functions are primarily seen as 'added to' the physical substrate, or as realized in physical objects, then the question remains how these functions are related to the mental states of human individuals, which, after all, form the core of the intentional conceptualization. If functions are primarily seen as patterns of mental states, on the other hand, and exist, so to speak, in the heads of the designers and users of artifacts only, then it becomes somewhat mysterious how a function relates to the physical substrate in a particular artifact. But relating them is exactly what happens in the design of artifacts.

Despite its usefulness as a starting point for philosophising about the nature of artefacts and the knowledge required by designers to design fit-for-purpose artefacts, the 'dual-nature' model has been criticised for its restrictive nature. Mitcham (2002) critiqued Kroes and Meijers' limited description of artefacts as having a 'dual nature', arguing that the nature of artefacts cannot be restricted to a 'functional-physical' nature. In an attempt to address the restrictiveness of the 
'dual nature' model, Verkerk, Hoogland, van der Stoep and De Vries (2007) added a third dimension to the 'dual-nature' model by extending it with aspectual intentions comprising a triad of knowledge content applied by designers: aspectual intentions, functional intentions and physical or structural components. The addition of the 'aspectual' dimension to the characterisation of artefacts is valuable as it opens the door for a creative and realistic consideration of the multi-facet nature of artefacts as they are experienced and conceptualised in real life. However, this extended epistemological framework's prejudice towards designers' knowledge of artefacts results in limited guidance for educators to account for the complex process of connection making between the various knowledge components. In addition, as in the case of the 'dual nature' framework, Verkerk et al.'s (2007) framework also conflates 'function and behaviour'.

Compromising for the function-behaviour conflation and for the static approach to decision making tools, Gero and Kannengiesser (2004) proposed an alternative ontological model, which they call 'The situated function-behaviour-structure (FBS) framework'. They argue that models that conflate function and the behaviour expectancy of artefacts are reductionist. They also criticise models that do not allow for visualising and comprehensively describing the individual processes of dynamic and individualised transformation of information. They further find that models that do not provide for generic design domains are problematic. Instead, these authors' framework adopts a situated approach to design that recognises dynamic decision making as a result of the influence of external information entering the decision making process across all domains. As such, the FBS framework is an adaptation of classic information processing theory where internal processing and sources of information are central (Simon, 1996). Its value lies in its depiction of the broad situated cognitive dynamics involved in the transformation of information in the problem solving space. However, the limitation of this framework is found in its own reductionist consideration of the multi-facet nature of artefacts in considering functionbehaviour as characteristics of artefacts only. In addition, it fails to address the complexity of different types of transformation, including lateral and vertical transformations. Therefore, it does not provide sufficient guidance regarding making choices, and judgments and decision making where these types of transformations are central (Goel 1995, p.119).

Due to the limitations of the various ontological and epistemological frameworks used to research, educate and practice design methodology, much of what designers know, what they do, how they do it and why they do it thus remains unchartered. The primary reason for this failure is identified by Cascetta (2001p. 95-173) as their inability to account for the psychological mechanisms underlying complex decision making processes. As a result, design methodology is insufficiently informed and design education curricula's prescribed assessment criteria, standards and suggested teaching strategies then become unstable. In an attempt to fill this void, it relies on the philosophy of technology.

I argue that seeing and finding that the 'relationship' is in the 'eye of the beholder' - it is the designer who selects and decides which physical elements are most suitable to approximate the intention of the client. Therefore, we need to consider how designers in the real world make, interpret and apply the relationships in the artefacts that they conceptualise. We also need to consider that it is not enough to consider epistemological theorising to untangle this very complex ontological issue, as is indicated in the literature. 
It is this gap that the model proposed in this paper attempts to fill. Rather than opposing existing models, in this study, I combine and adapt useful elements from the various models described earlier. From Goel's (1995) psychological characterisation of the design problem solving space, I take the concept 'hierarchical abstraction'. Also from Goel, I take his lateral and vertical transformations as represented in the sketches of designers. From previous work in extended cognition (Haupt 2013, 2015), that resonates with Gero and Kannengieser's (2004) situated characterisation of the problem solving space, I apply the notion of the integration of internal and external sources of knowledge to understand the origin of thoughts and the direction of transformations. From Verkerk et al. (Verkerk et al., 2007), I take the idea of extending the characterisation of artefactual knowledge to include 'aspectual intentions'. Finally, I add my own conceptualisation of how aspectual intentions, functional-behavioural intentions, and their connections with physical elements of an artefact feed into a third type of intention, namely 'implementation'.

Using Verkerk et al.'s (2007) epistemological modelling of designers' knowledge about artefacts, I add the level of aspectual intentions. From Gero and Kannengiesser, I borrow the methodological concept of considering both the internal and external sources of information and processes when exploring the ontology of decision making and hierarchical thinking. Instead of using the word 'expectation' found in utility models, and that of Gero and Kannengieser, I use the term 'intention', which is in line with the philosophical roots of my theoretical framework, which is founded in information processing (Goel, 1995; Mitcham \& Holbrook, 2006). From Goel (1995), I borrow the concept of hierarchical abstraction, and from Drummond, I borrow theoretical concepts of adaptive decision making processes. My theoretical framework therefore consists of a variety of theories (Figure 1), which are bound together by the extended information processing system discussed in the following section.

\section{Theoretical framework}

In an extended information processing system where existing internal knowledge is synergistically integrated with external knowledge, and with information that is constantly emerging externally, designers use their verbal and visual representations to express the content of their thoughts and the transformation of these. These transformations encompass and are anchored in the intentions of their design briefs, which forms part of their input information. Abstract intentions, including aspectual and functional-behavioural intentions, frame their decision making process and can be observed in the duration of their thoughts, to which they pay attention throughout the entire design process. They use it to structure their given design problems and develop concrete solutions, which they transform into concrete solutions that are characterised by specificity. During this process, their interpretation of abstract aspectual intentions serves as an anchor for framing their entire decision making process. Through anchoring (Tversky \& Kahneman, 1974), and subsequent approximation of their interpretation of intended aspectual, function-behaviour and implementation outcomes, with their ideas of the physical nature of the designed artefact, they are able to make unbiased judgments and subsequent adjustments. 


\section{Hierarchical decision making}

Broadly speaking, decision making can be thought of as the processes of considering multiple alternatives, choosing the most suitable one for a context and within the boundaries set by the contextual constraints, and committing to a final decision. Hierarchical decision making implies a complex process of multi-directional transformations. During this process, designers concretise abstract concepts into commitment to a decision about what a physical structure or an envisaged artefact need to take on in order to achieve their given intentions, and therefore serve the purpose for which each was meant. In an extended design task environment (Haupt, 2015), the computation involved in these processes is visualised in Figure 1.

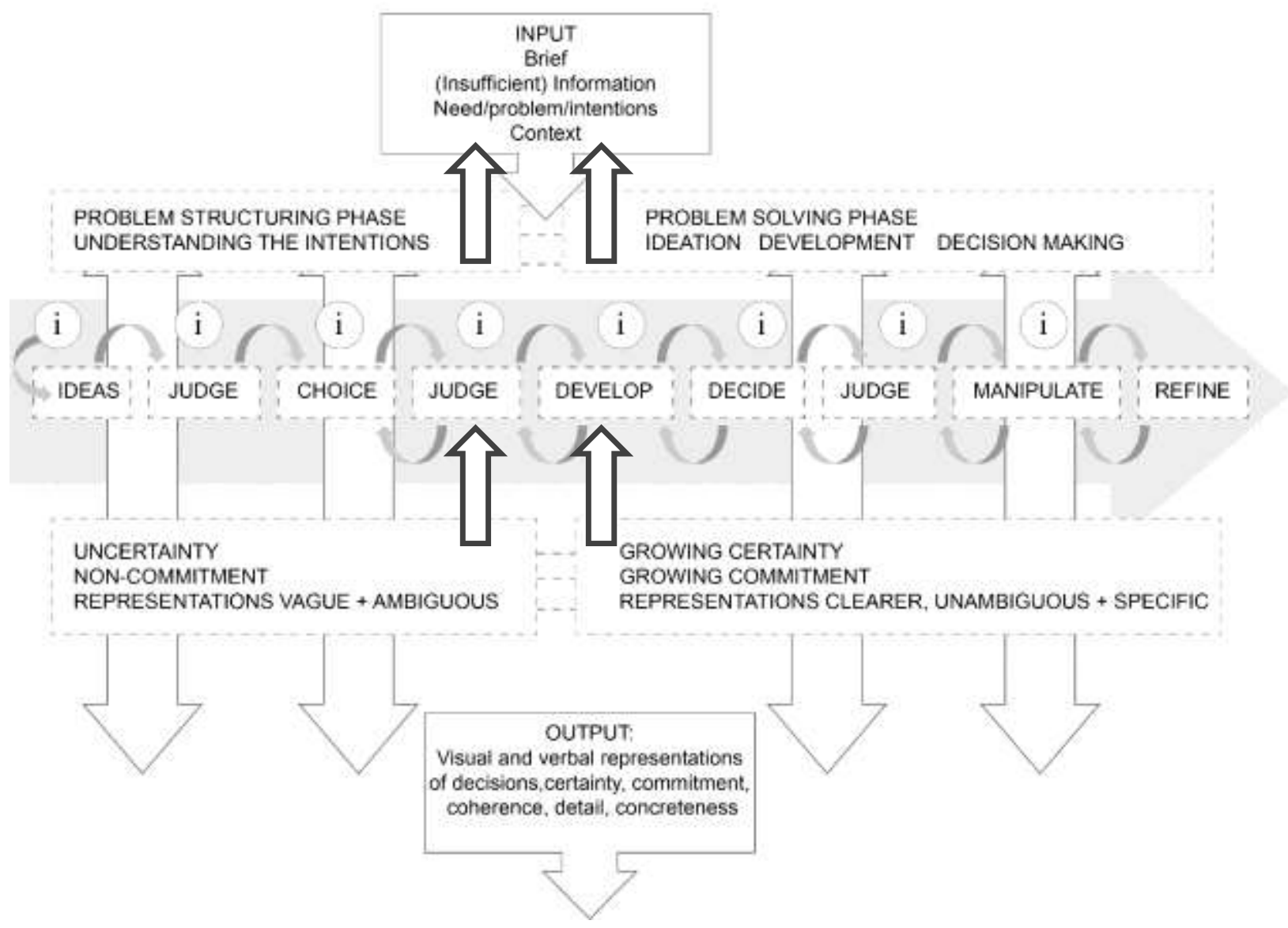

Fig 1. Computational actions involved in adaptive decision making

Input information can be sourced internally or externally right through the entire decision making process. Externally, the design brief that specifies the contextualised intentions through requirements and specifications are given to the designers, who internally process the implications thereof by connecting it with their existing domain specific, generic design knowledge and general multi-disciplinary knowledge. They fill in the gaps in their existing internal knowledge structure using existing knowledge recalled from their long-term-memories. They then combine this with external knowledge that they source from the external environment 
when and as they need it. These external sources include real objects, research from textbooks, the internet, specialist peers, and clients. Making decisions therefore relies on all these sources of information. It constantly, and in a non-linear manner, feeds into all the decision making actions including idea generation, judging by approximating ideas to abstract intentions that serve as mental anchors, making choices, developing ideas through hierarchical multi-directional transformations, making more judgments, manipulating representations to correlate with their judgment outcomes, and finally, refining their ideas/representations to correlate with increased approximation and specificity. This then ultimately results in a concrete modelling of their decision.

The distinguishable computation acts are often invisible, implicit and have to be derived from the representations of decision makers/designers. In these representations, it is possible to deduct a particular decision making act by distinguishing between basic cognitive operators, as summarised in Table 1.

Table 1

Characterisation of decision making actions

\begin{tabular}{|c|c|c|c|}
\hline $\begin{array}{l}\text { Decision } \\
\text { making } \\
\text { action }\end{array}$ & $\begin{array}{c}\text { Operator } \\
\text { (Information processing } \\
\text { theory) }\end{array}$ & $\begin{array}{l}\text { Embodiment principle } \\
\text { (Ecological theory) }\end{array}$ & $\begin{array}{l}\text { Level of } \\
\text { abstraction }\end{array}$ \\
\hline $\begin{array}{c}\text { Idea } \\
\text { (anchors and } \\
\text { approximation) }\end{array}$ & Propose & Intention-Attention & Vague \\
\hline Judge & Evaluate & Intention-Attention & $\begin{array}{l}\text { Vague } \\
\text { Specific }\end{array}$ \\
\hline Choice & Repeat, evaluate & Intention-Attention & Vague \\
\hline Develop & Elaborate, propose, & $\begin{array}{c}\text { Specificity } \\
\text { (adjustments) }\end{array}$ & $\begin{array}{l}\text { Vague- } \\
\text { specific }\end{array}$ \\
\hline Decide & Repeat, justify & Intention-Attention & Specific \\
\hline Manipulate & $\begin{array}{c}\text { Propose, evaluate, justify, } \\
\text { qualify }\end{array}$ & $\begin{array}{c}\text { Specificity } \\
\text { (adjustments) }\end{array}$ & Specific \\
\hline Refine & Evaluate, justify, qualify & Specificity & Specific \\
\hline
\end{tabular}

These operators and their connections with the decision making process assist in determining the efficiency of designers' decision making processes. The efficiency of the design process depends, inter alia, on the quality of the decisions designers take during the early phases of the design process (Goel, 1995). In turn, such quality depends on how close the selected solution concept approximates the intended outcome of an artefact (Gigerenzer \& Goldstein, 1996; Katsikopoulos, 2009). This 'proximity' therefore implies the coherence and correspondence mechanised by designers' internal gestalt formations (Wagemans, 2012), which in turn relate to their ability to anchor (Tversky \& Kahneman, 1974) their choices in their intentions. Therefore, 
'If a designer has particular intentions', the question is 'by what means does he/she achieve coherent decision making' and 'how can we trace the processes underlying such decision making?' These seem to be important questions. More importantly, however, we need to understand how designers achieve coherence in selecting and judging the appropriate physical elements decided on in order to meet their various intentions.

Bryan Lawson asks the general question, "How do professionals [...] get from their problems to their solutions?" (Lawson, 2006, p.8). In answering this question, Lawson (2006, p.136) says: "Through a clever mental process, some obstacle or conflict is simply removed by taking a particular view. In all such negotiation the skill lies in finding this view." One of the "clever mental processes' of expert designers is to 'find' an overarching 'philosophy' or abstraction (Level 1: abstract aspectual intentions). It is through such philosophy or abstraction that both the problem and the solution are framed (Schön, 1984) or anchored in (Tversky \& Kahneman, 1974), and through which internal consistency between intention and performance is achieved (Katsikopoulos, 2009). This finding represents a gestalt perspective on problem solving through global precedence (Haupt, 2015). Global precedence refers to the moment of insight at which designers 'frame' their problem solutions coherently by anchoring these in the relevant intention. In a decision making context, such framing refers to the instance where designers clearly understand the intentions of the design brief, and what is expected of the artefact that needs to be designed.

In order to understand the computational processes illustrated in Figure 1, these need to be analysed microscopically to do justice to the complexity of the process. It is not within the scope of this study to describe the intricate movements between the decision making actions within each hierarchical level. What I present in the following sections is a generalised description resulting from empirical evidence, with some examples, where relevant, to contextualise the theoretical elements of the proposed decision making tool, as represented in Figure 2. 
SOURCES OF INFORMATION:

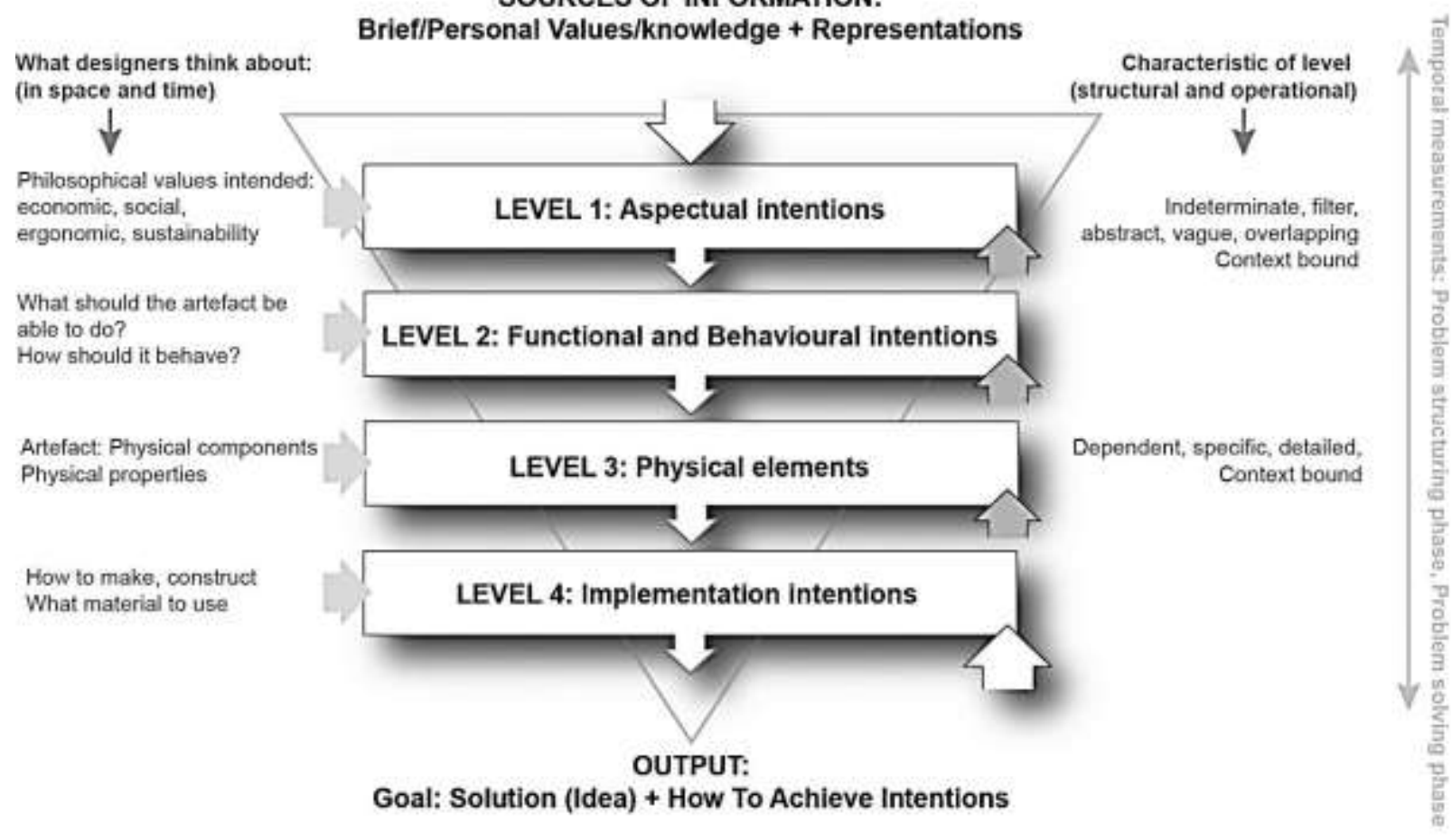

Fig 2. 4-level model of hierarchical decision making

Figure 2 shows the stratification of the thought content based on its abstraction-concretisation characteristics, and not on any chronological or priority order. 'Hierarchy' here refers to a hierarchical organisation of the transformation processes of vague intentions to a focused, concrete conceptualisation of the particular physical form, structure and properties of an artefact, as represented in the content of expert designers' thoughts. 'Abstract' here refers to a lack of detail and specificity. The model is therefore not a recipe for sequential computation, but rather a recipe for the methodology of considering knowledge about artefacts and coherent reasoning about contextual relevance and trade-offs when tracing the decision making process. These trade-offs form part of designers' judgment and evaluation processes, which can be observed in the multi-directional transformation structures of designers' hierarchical decision making processes Multi-directional transformations can be explained in terms of their lateral and vertical structures.

\section{Lateral-vertical structure}

The structure of 4-level hierarchical decision making implies two types of transformations: lateral and vertical (Goel 1995, p.119). These represent multi-directional thinking, and have been identified as an inherent characteristic of the hierarchical thinking of experts in the early phases of the design process (Haupt, 2015). 'Lateral' transformation refers to the movement from one idea to a slightly different idea. Designers generally engage in this type of transformation during their preliminary problem solving phase as it allows them to consider many alternatives. This is the phase during which designers do not yet commit themselves to a particular concept, but explore possibilities. Goel $(1995,119)$ emphasises that lateral transformation needs to occur in the preliminary phase of design problem solving. Lateral transformations tend to produce their 
own sub-sets of alternatives and elaborations thereof, which can be represented in orthogonal or tree-like sub-hierarchies, as illustrated in Figure 3.

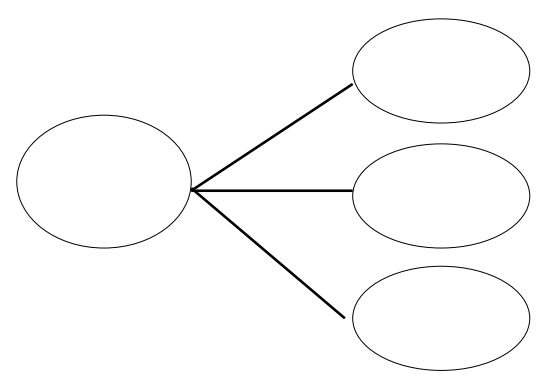

Fig.3 Lateral transformation structure

Laterally, developed hierarchies and sub-hierarchies are nested within vertical levels. They can be developed concurrently or independently of the relevant vertical level. They can be revisited, manipulated and expanded when necessary. The development of these is also dependent on the designer's ability to make associations and draw interrelationships with new information sourced internally or externally. Figure 4 is an example of lateral development seen in the alternative choices that have been generated around slightly different ways in which the same idea, boxes in this case, can be transformed into a storage system.

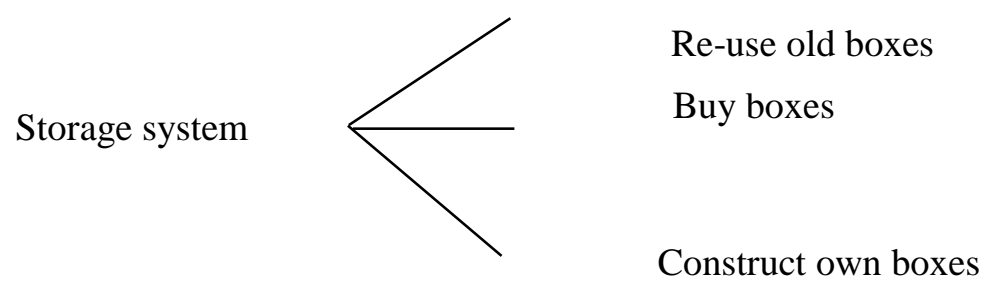

Fig.4 Example of lateral development of an idea

Figure 5 is an example of the lateral transformation seen in sketches made by PI in the $15^{\text {th }}$ minute of their protocol, early in their preliminary problem solving phase. 


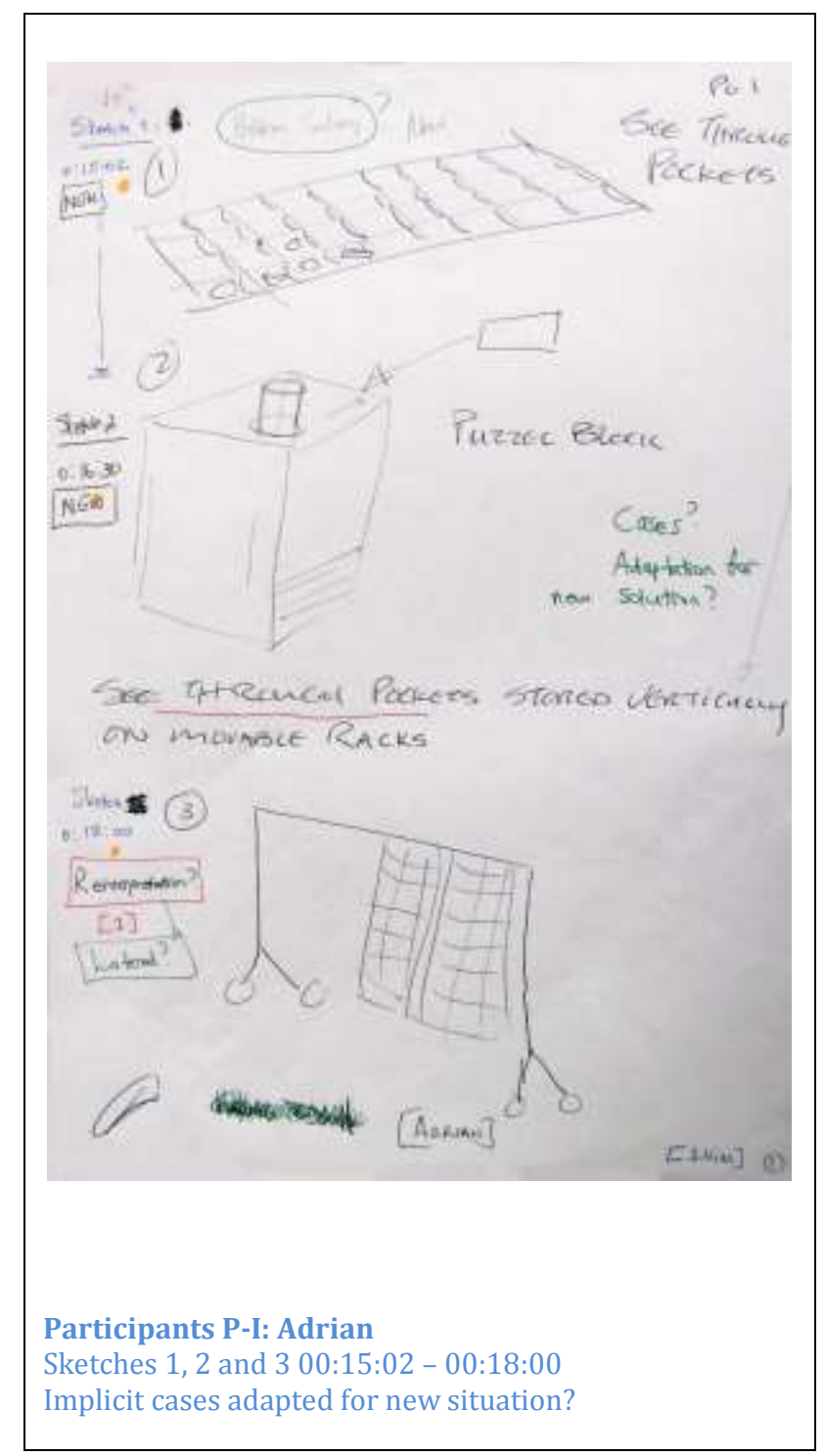

\section{Fig 5. Lateral transformation in sketches made by Participants P-I}

According to Goel (1995), good design practice is characterised by a balance between horizontal and vertical transformations. It follows that sufficient opportunities for judgment and decision making are provided to allow for the generation of new concepts, attending to existing ideas in more detail, or the discarding of others. The influence of the availability of information, the determinate role of the constraints inherent in each of the levels, and the particular context of the design process in terms of the specificity aggregation on each level taken into consideration plays a significant role in the evaluation process.

'Vertical' transformation, alternatively, entails that designers conceptually move from one idea to a more detailed and specific version of the same idea. Therefore, they do not generate new ideas, but rather clarify and develop existing ones in greater detail by adding visual information. Vertical transformations typically occur in the refinement and detailing phase as designers start 
committing themselves to a particular concept. As such, vertical transformations are based on previous ideas where designers reinforce and develop these ideas by means of iteration. This involves judgment and decision making. As the term suggests, vertical transformations develop in a top-down manner, as illustrated in Figure 6.

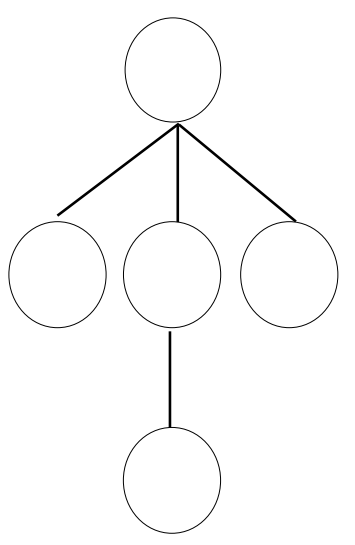

Fig 6. Vertical transformation structure

This means that, in vertical transformation, movement is made from one idea to a more detailed idea, treating the same idea with more specificity - a process that is dominated by intentiondriven thoughts and a negotiation of coherence and proximity. This direction of thinking is furthermore associated with the development phase within the bigger problem solving phase of the design process. This represents a commitment to a chosen idea that was generated in the horizontal transformations, and which is now developed in-depth after judging its appropriateness to achieve the set out intentions. The detail added consists of more exact ideas of the physical elements and their properties as means to achieve the required functionality, as can be seen in the example in Figure 7.

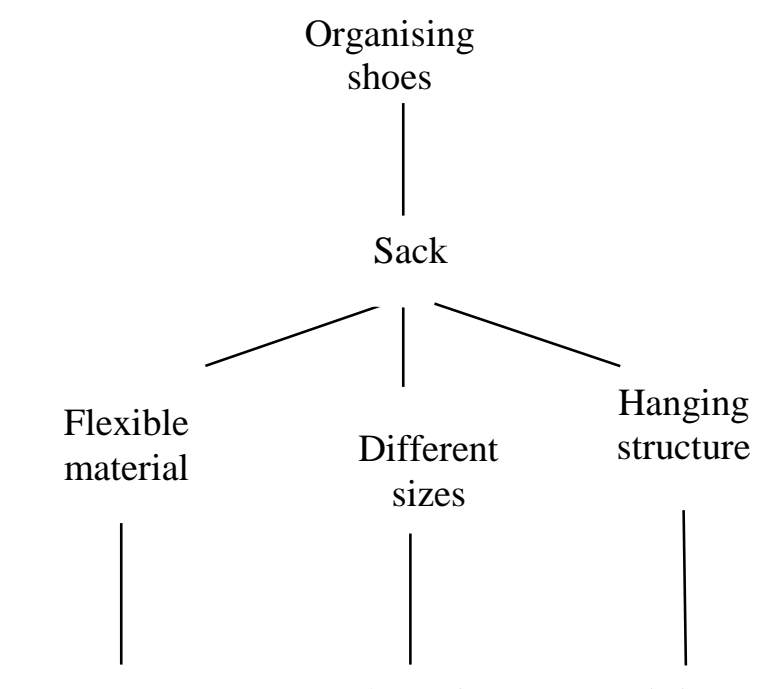

Transparent Three sizes Fit in car boot

Fig. 7 Example of vertical transformation 
Sketches made in vertical transformations are typically more exact and contain clear and transparent thinking and decision making. This transparency is a result of growing certainty of the conceptual connection between loose thoughts and internal coherence between the problem, intentions and detail ideas.

Operationally and theoretically, these transformations are intertwined, nested, interdependent, embedded and overlapping. Figure 8 illustrates how PI concurrently produced lateral and vertical transformations. Doing so enabled the industrial designers to compare ideas, something they could only do by visualising how each idea could work. This also allowed them to include both lateral and vertical transformation.

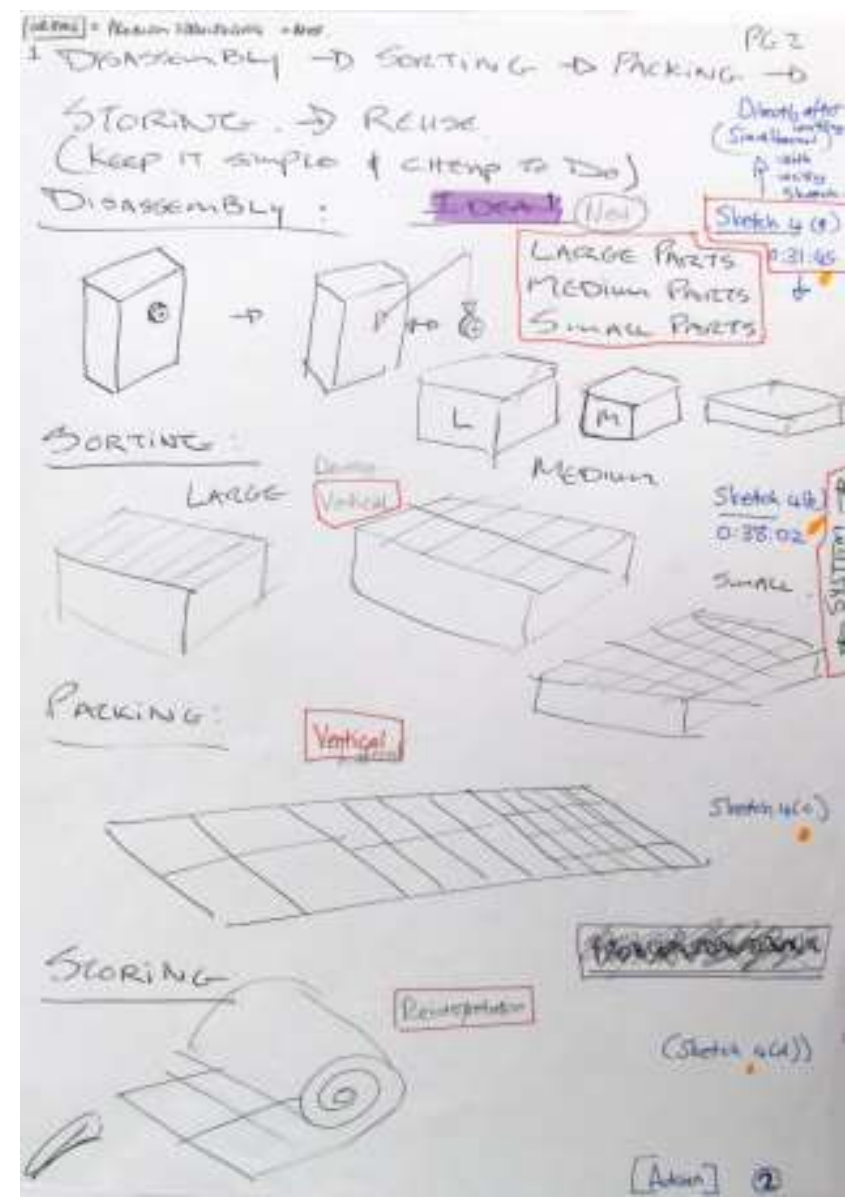

Fig. 8 Example of concurrent lateral and vertical transformations in one set of sketches produced by PI

Investigating these characteristics of hierarchical decision making is contextualised by the empirical findings of the protocol studies conducted on one pair of architects, mechanical engineers, and a team of three industrial designers. Each group received their own design task, which lasted two to three hours. The architectural task entailed designing an open air theatre on a university campus. The engineering task required the designing of a rotating platform for a theatre, and the industrial designing task entailed designing a managing and storage system for large quantities of $\mathrm{Lego}^{\mathrm{TM}}$ pieces used in an educational context. The tasks were considered 
complete once the participants decided on a particular solution to the problem. Concurrent with their verbal protocols, which were video-recorded, the participants produced rough manual sketches.

The study was conducted using mixed methodologies, with a qualitative dominance, due to an interpretivist approach. The data was coded according to a complex set of a-priori theoretical concepts representative of a critical realist approach. This was done to neutralise possible subjective interpretations. The study was guided by theories for extended information processing components. This paper focuses on the qualitative evidence of the original study.

In deriving patterns of abstraction, hierarchical decision making entailed the microscopic mapping of the dependency and interrelatedness of participants' thoughts, as indicated in Figure 9, which were derived from content analysis and data structuring. For the purpose of the current study, I draw from the qualitative data and results gathered from one group only, namely, the industrial designers (called 'PI'). It is easier to recognise and explain the transformation of one particular trail of thinking than to present isolated excerpts from the different pairs of participants within the constraints of this study. Selecting them was a random decision, and although the engineers displayed individual differences in the detail of their transformation processes, the general pattern of hierarchical decision making was representative of the entire sample.

Various sets of data structuring were used to arrive at abstract hierarchical decision making maps for the participants. The first set focused on sources of information, direction of transformation, and content of thoughts, which are visualised in Figure 9. 


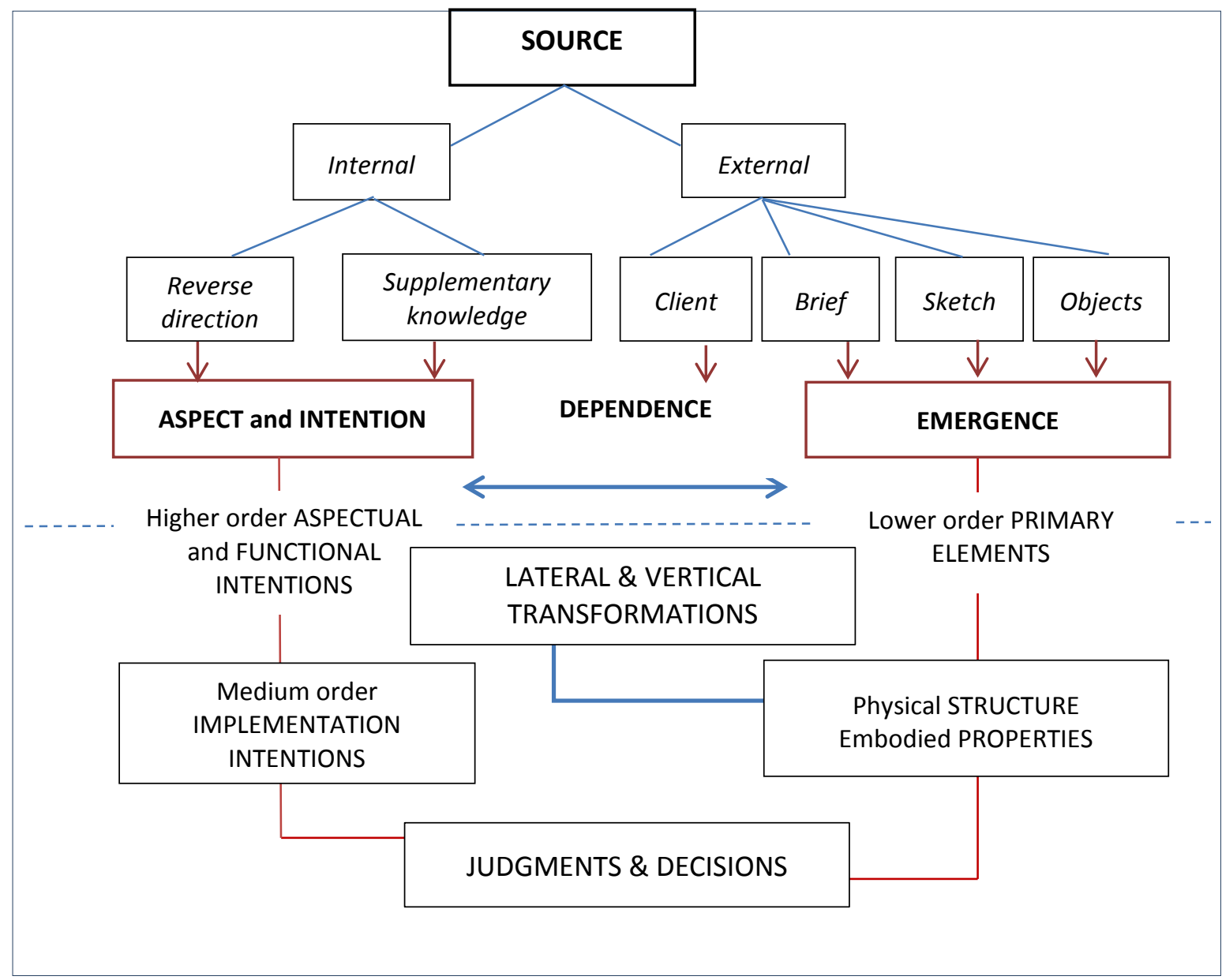

Fig. 9 Data structuring 1 for constructing hierarchical decision making maps: sources of information, direction of transformation, content of thoughts

As a second strategy, I determined the duration of time that the participants paid attention to particular ideas, which indicated to me where to look for commitment to an idea. Concurrently, I looked for correlations between verbal and visual data. When the participants developed sketches of something they had talked about, it was an indication of growing commitment. The third strategy for coding was to correlate duration with specificity and intention-attention categories set focused on the abstraction level within instances of abstraction-specificity and commitment (Appendix 2) when incrementally developing their designs.

From the psychology of design thinking, I used the notion of mental operators, which I adopted from the Information Processing Theory (Goel, 1995) and embodiment principles selected from Ecological Theory (Anderson, 2003; Gibson, 1986). This was done to individuate each mental activity or computation implied in the decision making process. From instances of identifiable idea generation, choice and deciding actions, it is possible to connect to the level of abstraction seen on a vague-specificity continuum, as explained by Goel (p.214). As can be seen from this characterisation of the decision making activity, summarised in Table 1, much overlap exists between the decision making actions, operators, embodiment principles and levels of abstraction. 
Based on the findings and their support in the literature, and within the context of the industrial designers' particular design task, I unpack the four levels and their implications for the hierarchical decision making processes in the following section. Their task was given as follows: 'Conceptualise a system that can identify, count and sort various Lego pieces available to Design and Technology Education students, which are to be used by lecturers and assistants to manage the storeroom. Lego is also used in remote venues during workshops and need to be transported to and fro.'

\section{Level one: Aspectual intentions}

The aspectual intentions, commonly referred to as 'design aspects' that designers consider are commonly agreed upon in design literature. These include abstract, non-defined principles embedded in aspects of aesthetics, formal elements, economics, materials, and manufacturing methods (Goel, 1995; Haupt, 2015; Oxman, 2002; Suwa \& Tversky, 1996). However, the mention of Level 1 intentions in the literature is generally integrated in discussions of the functional or behavioural statements of designers. In contrast, in this paper, I consider design aspects as a separate abstraction category of things that designers think about. I thus adopted Verkerk et al.'s (2007) inclusion as a separate, valid category of intentions in an epistemological sense. Here, I argue that the consideration of aspectual intentions serves an important cognitive purpose as it integrates and focuses the judgments and decisions that designers make around a central driving intention, which supports coherence together with functional-behavioural intentions. As such, it serves as an overarching directional or conditional category, which bridges the epistemological gap between functional and behavioural intentions, and the selection of physical components. This enables the implementation of thoughts, and is therefore on the highest, yet most abstract level of the hierarchical structure.

An aspectual framework that was defined by Dooyeweerd, and which was summarised by Basden (2000), consists of 15 multi-disciplinary themes or modalities governing our everyday lives. In this paper, I refer to these as aspectual intentions (Basden, 2000). This implies conceptual knowledge as well as normative judgements, which in turn means that the aspectual level serves as a filter providing constraints, and mechanising designers' control structures. Aspects play a deterministic or normative role, are irreducible, and all aspects contain echoes of each other (Basden, 2000). Aspects are, by implication, vague and general statements, and are sparsely distributed over the design process, although these tend to occur more often during the problem structuring phase (Haupt, 2013).

The aspectual intentions resonate well with conventional scientific disciplines that are related to aspects of quantitativeness, spatiality, kinematics, physicality, biotic/organics, sensitive/psychic, analytics, formativity, linguistics, social organisation and processes, economics, aesthetics, juridical, ethics/attitudes, and finally, the pistic/faith categories. The underlying assumption of this framework is that each aspect has its own set of laws and is irreducible, but may be transgressed (Basden, 2000). This implies that when designers refer to a particular aspect, their knowledge of the laws of a particular discipline is often implied and not necessarily explicated. Designers choose when to apply and pursue particular aspects, and when to contravene them without rejecting their validity. It furthermore implies that aspectual intentions serve as a topdown idea-generating mechanism, guiding designers' connection making with functional 
(behavioural) intentions and their selection of physical components and properties. Signifiers of aspectual intentions are typically found in each level. They are embedded in the choices that designers make on each of the other levels. It is context-bound and cannot be isolated from the lateral and vertical transformations, and is therefore open to different interpretations.

In the protocol of PI, it became evident that they considered the aspectual intention of the economic aspect, expressed as 'simplicity', which was in breach with what the design brief required. However, their strong belief in the value of such intention was confirmed throughout their entire protocol. Although the industrial designers generated a solution that complied with the expectation of a self-sorting device that could satisfy the client's requirements, and they dwelt on the thought for a relatively long time, they questioned the appropriateness of a complex device functioning in the way that their design brief suggested. An example of such embedded aspectual intention is evident in the following excerpt:

\section{BRIAN 00:31:03 \\ My thought also go a bit well, what is the simplest possible solution with the least, uhm ... parts and material and in fact should one just stick with this purely manual system what they have, but they employed people to do it. Systems ...}

The excerpt shows the abstract nature of their statement, as seen in the words 'purely manual system', which points to simplicity. It contains no details provided yet, however, reference to employing people to do the work instead of designing a machine that can do the work on its own shows the overlap between aspectual and implementation intentions. Their vertical transformations are evidence of this commitment, as seen in the growing specificity of their idea (see Figure 11 further on).

They pursued their aspectual intentions of 'economics' and 'efficiency'. These were seen in the chunks of long duration, which indicated their commitment to their intentions. Table 2 shows the role of the global precedence that the aspectual intention level plays in the way in which it permeates all four levels of their hierarchy of decision making. This ensures coherence in lateral and vertical transformations, as seen in the long and short chunks of attention to one theme displayed in the excerpt from the participants: 


\section{Table 2 Participants P-I's aspectual intentions permeating all four levels of hierarchical decision making}

\begin{tabular}{|c|c|c|c|}
\hline \multicolumn{4}{|c|}{ LONG CHUNKS of ATTENTION } \\
\hline $\begin{array}{l}\text { Aspectual } \\
\text { intention }\end{array}$ & $\begin{array}{l}\text { Functional and behavioural } \\
\text { intentions }\end{array}$ & Physical elements of objects & Implementation intentions \\
\hline $\begin{array}{l}\text { Economic } \\
\text { Physical }\end{array}$ & $\begin{array}{l}\text { - } \text { - Corting } \\
\text { - Saving space } \\
\text { - Allow discovery } \\
\text { - Easy access to Lego }{ }^{\mathrm{TM}} \\
\text { - } \text { pieces } \\
\text { - } \text { Allow for various sizes } \\
\text { - Easy transportation } \\
\text { - Weighing } \\
\text { - Controlling access and } \\
\text { - } \text { recovery } \\
\text { - Dasy to carry } \\
\text { - Opurable } \\
\text { - Easy disassembly } \\
\text { - Easy recovery } \\
\text { - Easy sorting }\end{array}$ & 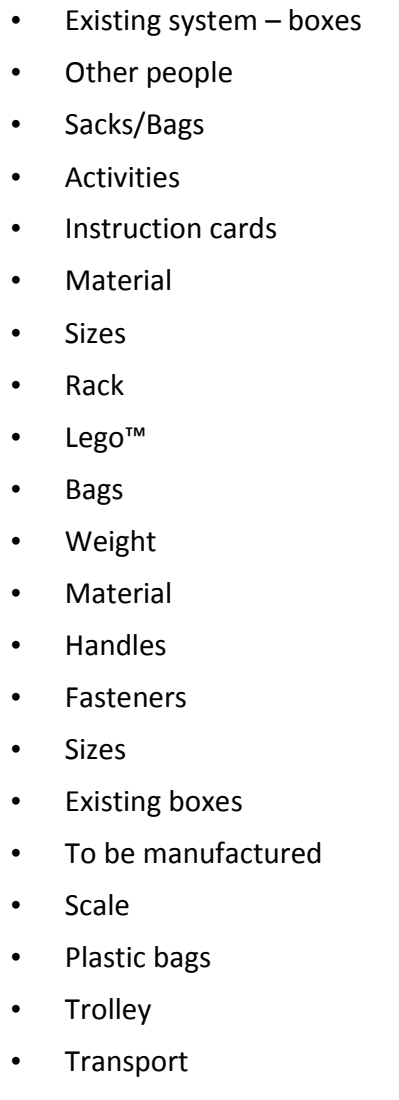 & $\begin{array}{l}\text { - Use the least parts and } \\
\text { material } \\
\text { - Stick with manual system } \\
\text { - Employ people to do the } \\
\text { work } \\
\text { - Change approach: Use } \\
\text { existing manual system. } \\
\text { Reorganise sequence of } \\
\text { activities } \\
\text { - Students to form part of } \\
\text { management activities/ Put } \\
\text { on scale Maximum weight } \\
\text { per bag } \\
\text { - Weigh - not count } \\
\text { - Organise according to size } \\
\text { of Lego'm pieces: large, } \\
\text { medium, small } \\
\text { - Allow for visibility } \\
\text { - Clear front } \\
\text { - Existing A4 paper boxes } \\
\text { - Plastic bags in boxes } \\
\text { - Weighing part of situation } \\
\text { Involve students } \\
\text { Employ other people }\end{array}$ \\
\hline \multicolumn{4}{|c|}{ SHORT CHUNKS of ATTENTION } \\
\hline Economic & $\begin{array}{ll}\text { - } & \text { Cost } \\
\text { - } & \text { Storage space } \\
\text { - } & \text { Labour } \\
\text { - } & \text { Sack } \\
\text { - } & \text { Digital management } \\
\text { device } \\
\text { - Flexible material } \\
\text { - Different sizes } \\
\text { - Draw strings } \\
\text { - Convert sacks into } \\
\text { working sheets on tables }\end{array}$ & $\begin{array}{l}\text { - Transport } \\
\text { - Storage } \\
\text { - Recovery }\end{array}$ & $\begin{array}{l}\text { - Use existing objects to } \\
\text { lower cost } \\
\text { - Employ people at low cost } \\
\text { - Reorganise sequence of } \\
\text { activities } \\
\text { - Vertical hanging system } \\
\text { working like shoe } \\
\text { organising. Need a clothes } \\
\text { line } \\
\text { - Different sized pockets } \\
\text { - Sacks of three different } \\
\text { - } \text { sizes } \\
\text { - Fit in car } \\
\text { - Involve users } \\
\text { - Use convertible and multi- }\end{array}$ \\
\hline
\end{tabular}




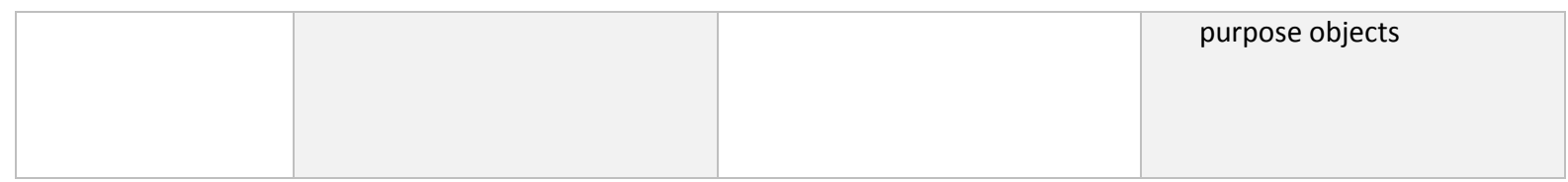

The second level on the abstraction hierarchy is functional behavioural + intentions.

\section{Level two: functional + behavioural intentions}

Thinking about and researching Level 2 thoughts, namely, functional + behavioural intentions of artefacts, has been the topic of many studies (Kroes, 2002; Mitcham, 2002; Verkerk et al., 2007). Designers receive some required expectations regarding the designed artefact's function and behaviour in their design briefs, which they incrementally refine as their understanding of the context develops (Goel, 1995, p. 106). Here, designers attempt to get a clear picture of what the purpose of the required artefact is in the context of the given problem. They address the insufficiencies in the design brief regarding context in order to derive the functional requirements expected to be achieved, as articulated in the design briefs. Typically, they would ask 'what is this machine supposed to do? Where is it going to be used? Who will be using it?' Closely related to this type of questioning are the following questions: 'What should it be able lift? How much weight should it carry? At what minimum and maximum speed should it be operating? Under particular conditions should it be able to perform efficiently?' This means that designers think about the function and behaviour of an artefact concurrently, and thus they tend to conflate statements about functional intentions with those of the expected behaviour of envisaged artefacts or their individual components. Functional goals can be formulated and reconceptualised throughout the entire design process. The temporal and spatial distribution over the entirety of the early phases, however, is denser than that of aspectual intentions, as indicated in Table 2. Designers typically return to this level of thinking repeatedly as they connect functionality with aspectual intentions (Level 1), and ensure internal coherence with their choice of physical elements (Level 3). Designers spend more time and longer periods on attending to functional intentions when they are committed to a particular aspectual intention (Table 2).

However, functional thoughts are more specific than aspectual intentions are generated, an observation emphasised by Goel (1995). He ascribes this trend to the characteristic incremental solving of design problems, which means that designers discover sub-problems as they go along, with the emergence of new functional intentions throughout the process. Prolonged time is also a result of the availability of information, and its conflation with behavioural thoughts and preliminary judgements.

As Level 2 intentions are usually prescribed by the client via the design brief, designers tend to develop them vertically in a top down-manner. However, the results of their protocols have indicated that they primarily think about the physical elements first, and then judge their functionality and subsequent behaviour. Early on in their protocol, they generated multiple lateral ideas about alternative physical concepts without connecting these with functionality. This tendency is well documented in the studies of Suwa, Purcel and Gero (1998), and Cross (2001). To me, this indicated a lack of commitment at that stage to any of these physical elements until they had constructed Sketch 3 and 5. It is in these sketches where the inclusion of functional items occurred for the first time while articulating physical element thoughts on Level 4. Once they had connected function with physical elements that embed aspectual intentions, the designers' conceptualisations became much more detailed and specific than in the case of 
fragmented ideas without such connection. An example of trying to understand the function of an artefact and its context is illustrated by Figure 10. Participants PI used their sketches extensively to facilitate their understanding of functional and physical connections and diagrams to evaluate their conception.

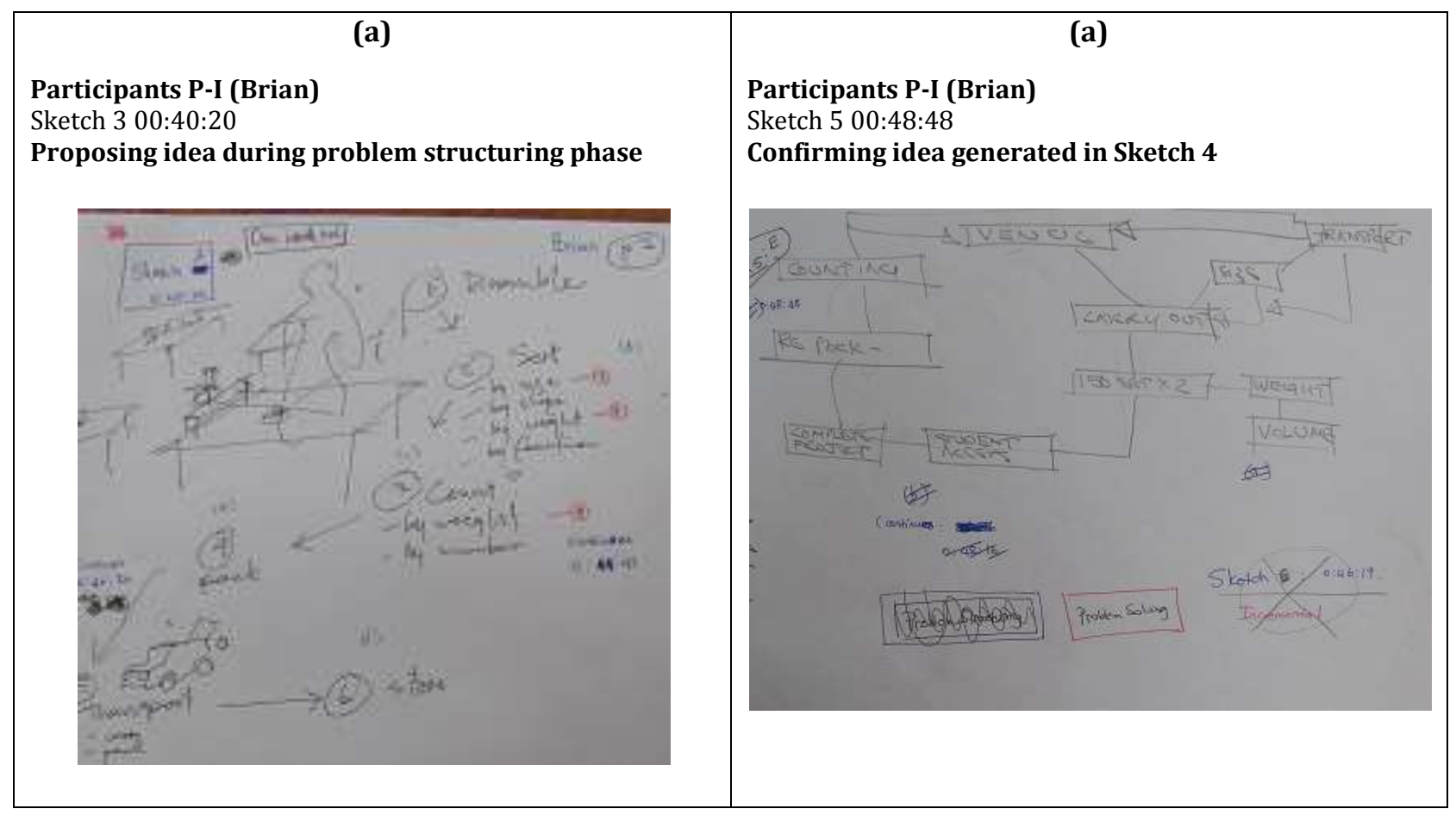

Fig. 10 Visualisation of functional intentions while articulating Level 4 thoughts

The lateral development of functional thoughts typically depends on information emerging from Level 3 information, and the designers' judgment of appropriateness. It is their consideration and elaboration of physical elements, including thoughts of the particular type of structure, individual components, material, and its internal and external properties that forces them to turn back to Level 2. Revisiting functional and behavioural thoughts is a bottom-up transformation as they judge the functionality and behavioural implications of each physical component. Yet, an interesting multi-directional determinism exists here. It is the aspectual intentions that a designer focuses on that seems to provide focus and overarching norms, which drive designers' philosophical value judgments regarding appropriateness. Alternatively, functional and behavioural intentions seem to drive efficiency value judgments.

\section{Level three: Physical elements}

Much has been researched and philosophised (De Vries, 2006; Kroes \& Meijers, 2002) about the physicality of artefacts. The third level relates to the basic materiality of artefacts, and their components and properties. Physical elements include ideas about objects on their own, e.g. 'trolley', or components thereof, e.g. 'handle', or 'wheels'. They may also refer to lower order primitives, e.g. shape, size, colour, texture, material, and spatial composition. Designers typically ask questions and generate ideas about what components a particular object, for example the forklift, might consist of, what its total size and its spatial and physical relations with its 
individual components should be, what size it should be, and what material it should be made of. Reciprocal thinking about each individual choice's connection with Level 2 and Level 1 thoughts occur concurrently with the horizontal generation of ideas about these questions. Figure 11 is evidence of extensive choosing between ideas, and the implicit execution of evaluation functions by judging externalised in the transformation sketches of PI.

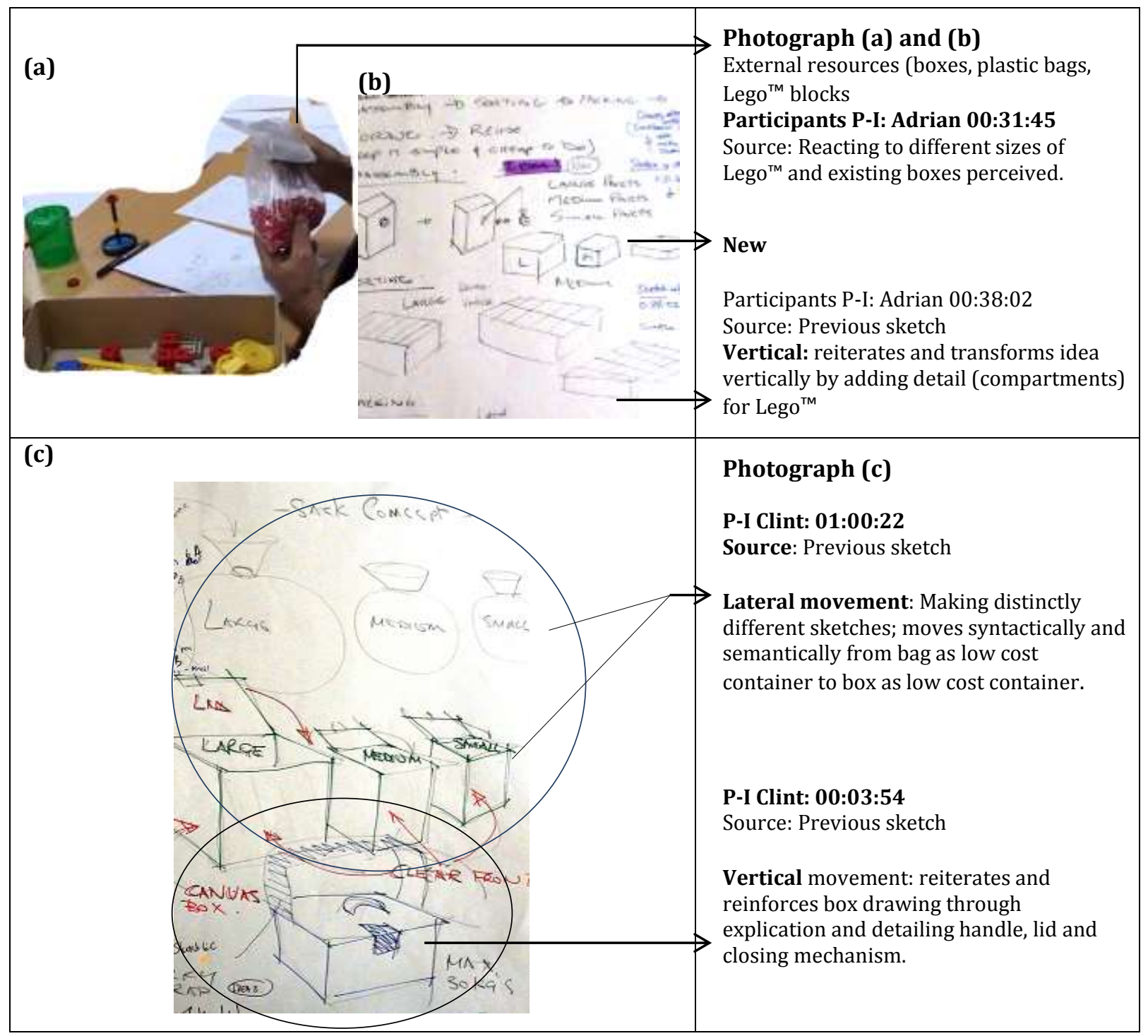




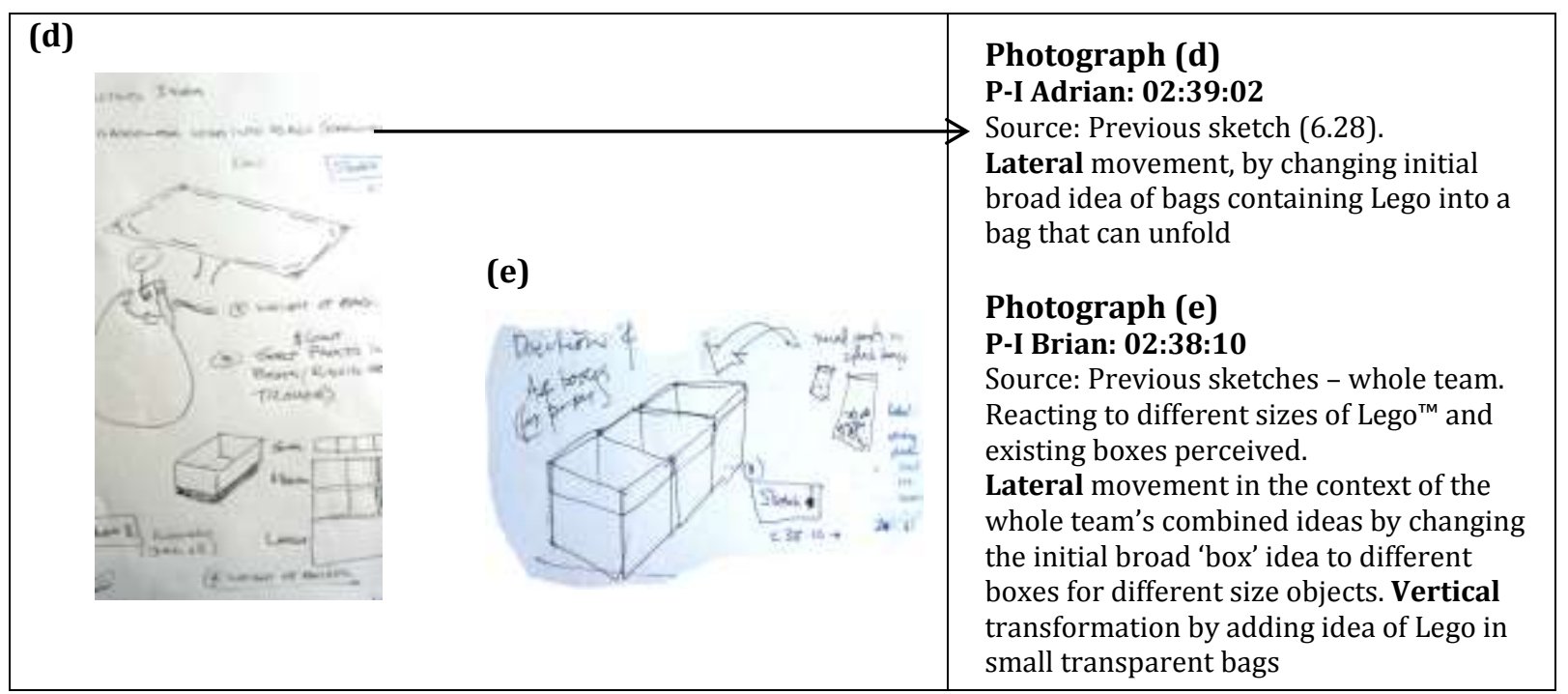

Fig. 11 Participants P-I lateral and vertical transformations

By implication, when designers generate ideas, judge, and transform their physical components of their artefacts vertically or laterally, the need for external visualisation is evident. In order to revisit, manipulate and refine their preliminary ideas, aggregation becomes more refined; detail and specificity levels are incrementally growing until initial dense, ambiguous representations become clear, transparent and accurate. Unambiguity develops into concrete physicality in the vicinity of some internalised representation of existing objects, and the association with their historical normative relations with functionality and behaviour (Level 2). Such development can be seen in designers' recall of previous cases that resemble similar relationships, also known as 'case immersion' (Goel, 1995, p.91). Designers' thinking about physical objects and their material elements are also seen to be influenced by perceptual referents in the external environment. This means that they take cues from external representation of existing or imagined objects from real, existing objects, as shown in Figure 12 further on.

\section{Level four: Implementation intentions}

Less has been written in design literature about implementation ideas, but it has recently reappeared in psychology literature (Brandstatter, Heimbeck, Malzacher, \& Frese, 2003). This is where questions are being asked about how designers' external situations, including the context and choices made about the physical nature of their designed artefact, influence their articulation in implementing their aspectual and functional intentions to achieve the desired behaviour. Making implementation functions is therefore purely self-initiated, and a result of previous commitments. They are more specific than aspectual and functional levels as this level is closely related to designers making and propagating commitment (Brandstatter et al., 2003; Gollwitzer \& Schaal, 1998). As designers incrementally develop their ideas (Goel, 1995), implementation intentions serve as the saturation point of the hierarchical model. In Figure 12, it is clear how PI used both sketches and writing to clarify their implementation intentions. 


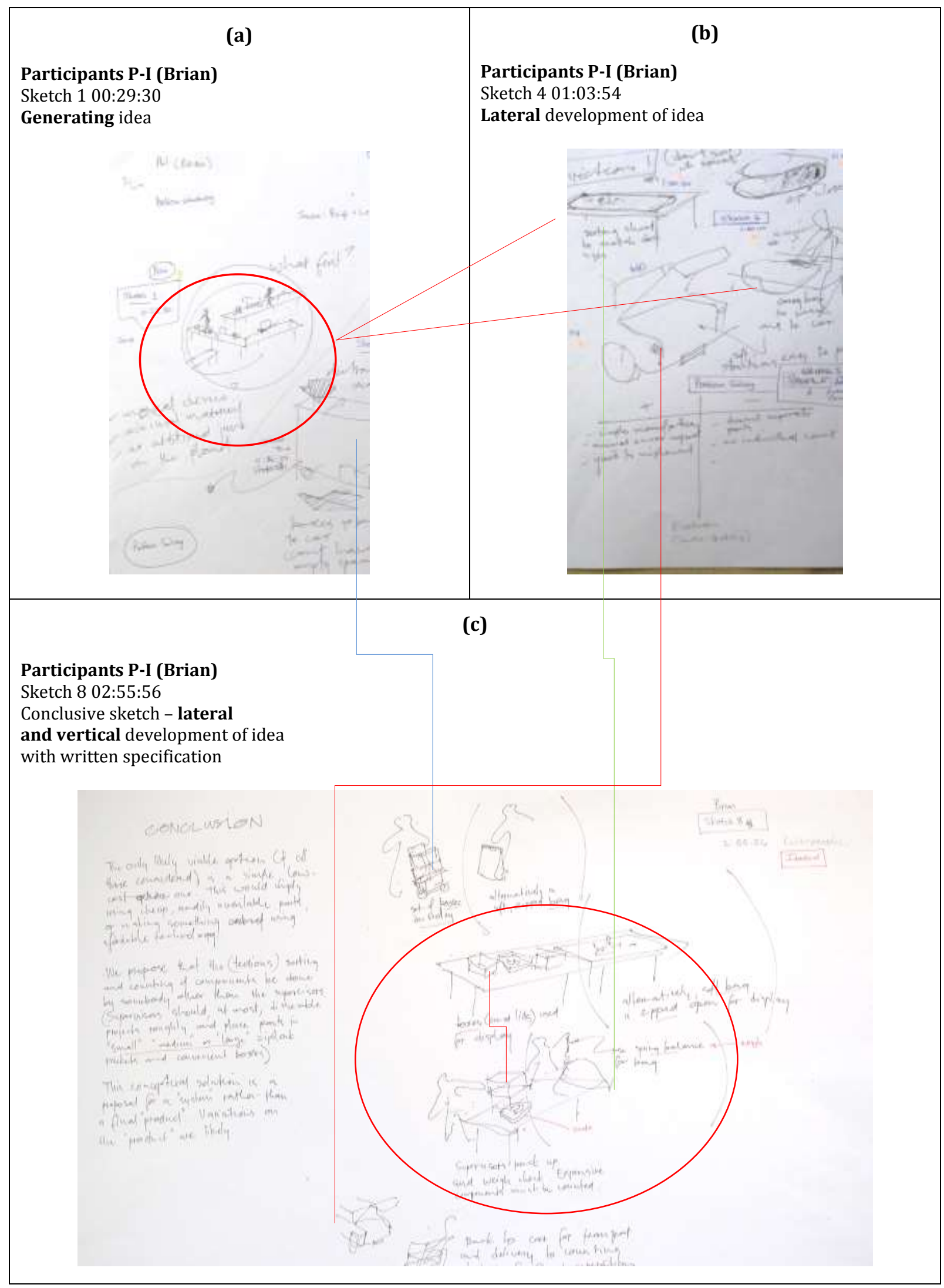

Fig. 12 Participants P-I's visualisation of their implementation intention 
The sketches and notes written by Brian demonstrate PI's commitment to their idea of adapting the existing storage and management system consisting of boxes. They did this by changing the sequence of events and involving students to help lecturers manage their use of Lego ${ }^{\mathrm{TM}}$. Their sketches suggest their understanding of educational procedures in terms of the students participating in the control and management of the $\mathrm{Lego}^{\mathrm{TM}}$ that they will use as a learning aid.

The content of Brian's additional notes on the sketches suggests his aspectual consideration of economic design, which is not visible in the sketches captured in Figure 12. The vague shapes in these sketches were developed laterally, as demonstrated in the sketch captured in Figure 12 (b). The basic rectangular shapes represent tables, and rough sketches visualise people moving and participating in unpacking, standing at tables, and rolling and unrolling cloths that serve as containers. Brian included a rough sketch of a motor vehicle with an open boot, which suggests transportation as a functional intention. In Figure 12 (c), the detail in the sketches suggests lateral and vertical transformations. The vertical transformations are embodied in the sketches, which included boxes stacked onto each other and loaded on a trolley. This indicated their implementation intention. Brian's repetitive visual reference to 'motor car' alluded to his commitment to the concept of a storage system that could fit in a motor car. He also repeatedly sketched how the relevant people might use the artefacts (boxes, tables, trolleys) involved. Therefore, showing the interaction between objects and people became a way of expressing PI's implementation intention of involving users in the management of the Lego ${ }^{\mathrm{TM}}$. In the case of Brian, it did not seem to be the inclusion of detail that expressed commitment, but rather the repetitive use of the same physical elements.

\section{Discussion}

From the literature, and empirical evidence provided here, it is apparent that hierarchical decision making is complex and requires multi-directional thinking. This enables designers to adapt to constantly changing situations within their own design processes. In constructing decision making tools that foster cognitive abilities similar to those of experts, this requires an approach that allows for complexity, openness and non-linear thinking. What furthermore became clear from the evidence provided here is that decision making is not a single event where designers 'see the light' and suddenly 'get' the solution. Instead, it is a process involving lateral and vertical transformations of their ideas during which they make judgments, choices and decisions while concurrently evaluating, manipulating and refining these. While they move in this multi-directional manner between ideas, they incrementally make connections between abstract aspectual and functional intentions, and physical elements affording behaviour that approximates these intentions. They make these internal connections by actively and synergistically integrating their domain specific and generic multi-disciplinary design knowledge with their own representations. They also make these connections by perceptually interacting with other external sources of information. The end product is an increased clarity about the unique physical form that their design artefact should have in order to achieve the given abstract intentions provided in their design briefs.

The case study presented earlier demonstrated that it is possible to gain relatively direct access to seemingly 'invisible' cognitive processes. Tracing hierarchical decision making, in particular, becomes 'visible' in the sketches of designers. Instead of only analysing verbal utterances, the content of their thoughts, as well as the connection making process becomes detectable. This 
proves the value of sketches for educators as a source of information about their students' cognitive state when negotiating decisions.

Implications for hierarchical decision making tools

From the literature, and empirical evidence discussed earlier in the case study, the following principles should be embedded in the design of a representative hierarchical decision making tool:

1. Allow for development of a mental representation of the environment/context where the problem is to be solved (verbal and visual).

2. Account for competence in drawing relationships, pattern recognition, and association (lateral and vertical).

3. Allow for updating (result of reflection, evaluation)/manipulating the representation in order to align it with the current state of the situation.

4. Encourage the use of tools that should be available and used to narrow down the area of active interest in the knowledge domain and to render it coherently with the problem setting - this implies the availability of internal and external tools (which is not the focus of this study).

5. Account for mentally modelling how the environment will change as a function of the decision taken.

6. Encourage mental testing of alternatives on the basis of a set of criteria such as appropriateness, area of influence, consequences, space and time allowed to correct, and/or change a decision if it turns out to be wrong.

\section{Transfer of hierarchical decision making}

The hierarchical transformation of abstract intentions to concrete, appropriate designs for artefacts should be encouraged. It is thus necessary to consider the influence of the sources of information on the hierarchical structuring of knowledge acquisition and processing during the early phases of the design process. This requires close cognitive coaching by the educator through cognitive apprenticeship.

Cognitive apprenticeship is not a new educational concept, but was applied in traditional craft and industrial trades. It promotes the learning of processes that experts use to handle complex tasks. However, traditional apprenticeships were directed at the external outputs and physical skills of making and crafting. Cognitive apprenticeship is a development of this concept (Collins, Brown, \& Newman, 1987). It means that the unseen internal intellectual processes are accessed through evidence of understanding, knowledge and processing in external representations such as words, sketches, and gestures. The role of the educator is therefore one of 'mimicking' expert design thinking. Educators further need to coach the thinking skills involved in solving design problems and closely guiding students, through instruction, regarding the acquisition and interpretation of information during investigating tasks. It is in these tasks that learners need to solve design problems when they design artefacts.

In essence, apprenticeship emphasises methods for carrying out tasks in a particular context. This requires specific knowledge, for example, generic design knowledge connected with 
contextualised domain specific knowledge. Apprentices are known to learn specific methods, including observation, coaching and practicing the methods that the mentor models and coaches them in (Collins et al., 1987, p.2). In order to be effective, apprentices repeatedly observe the master executing the desired process, which typically involves many different, but interrelated sub-skills. The apprentice then attempts to execute the process without the guidance and help of the master. Key to meaningful coaching is scaffolding, one of the founding cognitive tools adopted by constructivist educators who have a situated view of learning (Petrina, 2007, p.176). This means that educators need to systematically and incrementally contribute to the nature of the knowledge that learners acquire from understanding the design problems they need to solve. Learners also need to know how to proceed in solving a problem in its complex broad social context, as well as relevant sub-skills (Collins et al., 1987).

In order to transfer the skills of connection making into meaningful learning experiences that resonate with decision making activities, cognitive apprenticeship seems like a suitable pedagogy. Cognitive apprenticeship bridges the traditional gap between educator centred and learner centred approaches (Ertmer \& Newby, 2013) It furthermore has the advantage of providing guided instruction while controlling learners' phases of self-regulation during their decision making processes. It does this without reducing their transition into independent 'designers' within the boundaries of their particular developmental phase. This advantage could compensate for some of the limitations of the constructivist approaches, as criticised by Kirschner, Sweller and Clark (2006). This is based on the limitations of unguided instructional methodologies. These include approaches such as discovery learning (Anthony, 1973), problembased-learning (Savin-Baden, 2007), situated learning (Robbins, 2009), and inquiry-based learning (Edelson, Gordin, \& Pea, 1999), which is often applied in technology education (Johnson \& Daugherty, 2008; Petrina, 2007).

Conventional methods of teaching technology, which are based on general traditional apprenticeship methods, tend to better promote the learning of conceptual and factual knowledge through isolated activities and tasks (Collins et al., 1987). Other traditional methods found in cognition-related studies in technology and design contexts, as reported by De Miranda (2004), and the best practices documented in De Vries, Custer, Dakers and Martin, (2007) collectively point to the ineffectiveness of educator-centred approaches. They also allude to the ineptitude of isolated tasks and activity based strategies in fostering real world expert design problem solving behaviour. I support Hennessy's (1993) criticism of constructivist theories, which assumes that novices automatically learn through approaches such as situated cognition. Hennessy finds these to be unrealistic and non-contributory to effective learning, as is advocated by some authors (Ertmer \& Newby, 2013). Instead, Hennessy pleads for the application of cognitive apprenticeship as preferred pedagogy in technology education as it requires the active cognitive involvement of the educator with the thinking progress of students, which ensures meaningful learning experiences.

Design projects, if taught in this manner, can contribute to conceptual knowledge being transformed from abstract information to concrete, usable entities of knowledge. Such knowledge is used in a variety of sub-tasks that are relevant to the distinct cognitive phases of the design process. These have the potential to encourage a deeper understanding of the meanings of the concepts. Rich networks of memorable associations between essential working 
principles embedded in the conceptual knowledge of technology, for example, in particular types of structures, materials processing, mechanical and electric/electronic systems can be formed. Collins et al. (1987, p.3) emphasise that the focus of learning-through-guided-experience is on cognitive and metacognitive processes, rather than on physical skills and processes, which can be achieved through a technique called 'reciprocal teaching'.

The choice of connecting guidance for technology education to a Theory of Cognitive Apprenticeship, as interpreted by Collins, Brown and Newman (1987), although not new, is still relevant (Hennessy, 1993). This is the case due to its historic roots in guiding learners to acquire knowledge and experience in carrying out complex or realistic tasks. Furthermore, it is considered to be a teaching strategy that facilitates the embedding of learning of skills and knowledge in the social and functional context of their use. Technology curricula's design projects, which provide a socially situated design problem to solve, are therefore ideally connected to the application of the cognitive apprenticeship approach to teaching and learning.

Finally, the theory assumes that accessing unseen cognitive processes by studying external representations of internal thoughts and processes is closely related to the meta-theory of cognitive science. This meta-theory focuses on control systems and feedback loops when processing information during problem solving activities. The implication for teaching and learning the design process in technology classrooms is therefore that of reciprocity. This means that what the educator represents to learners as 'knowledge', 'information' and conceptual understanding needs to be clear and unambiguous so that learners are clear about the meaning and content thereof. Learners should then also be able to connect it to their design briefs. In turn, learners' reaction to instructions in their own external representation, including their sketches, spoken and written words, demonstrations and gestures, becomes an external indication of their interpretation and application of knowledge in investigating and designing tasks. Finally, these externalisations need to be fed into the system of information processing by comparing, critically evaluating and amending their investigative insights, and any ideas proposed during their designing activities. This should be done to constantly develop a much focused alignment between the intentions of the design brief and the ideas represented in their externalisations.

Although cognitive apprenticeship theory on its own does not account for external representation comprehensively, it does seem to have the potential to allow for its effective integration in teaching and learning in decision making learning contexts. It allows for reciprocity, which implies that learners (and educators) constantly refer back to the content of their spoken and written words, as well as their sketches, and evaluate its relevance to the intentions of their design problems, which is advantageous. Furthermore, the intrinsic coherence between the networks and interconnections made during the entire design process provide educator-regulated, as well as learner-regulated opportunities for evaluating coherence. From the educator's side, it requires intimate and critical involvement in the content and structure of learners' spoken and written words, their conceptual and developmental sketches, mind maps, flow diagrams and physical demonstrations or gestures during the entire design process. This needs to be checked against each of the hierarchical levels outlined in Figure 2. It furthermore requires educators to demonstrate to learners what it means to think in a 'designerly way' through scaffolding, questioning and demonstration. From the learners' side, it requires intentional application of conceptual knowledge, recognition of relevance, and metacognition informing them of what 


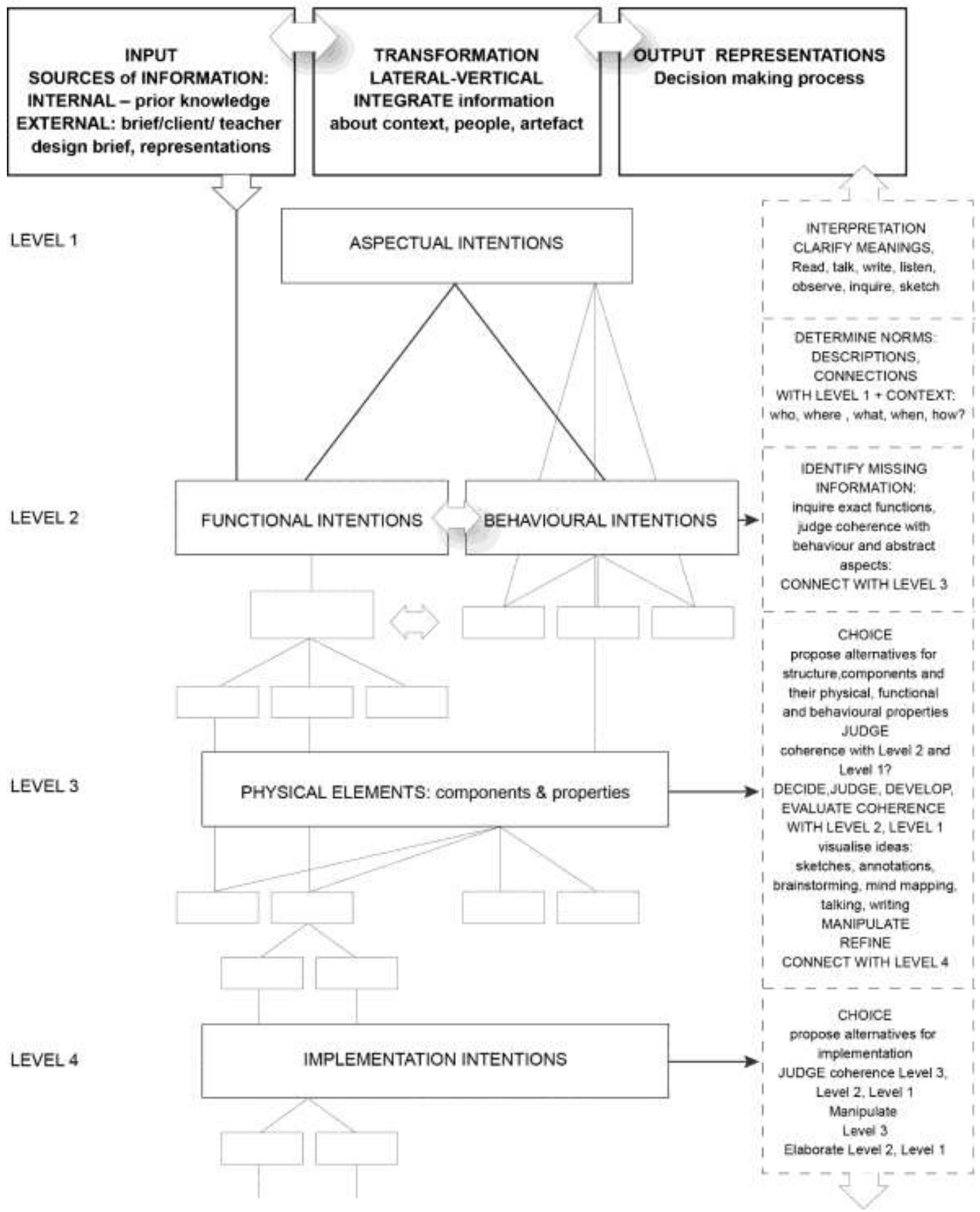

Fig. 13 Hierarchical decision making tool 
information they still require on each of the hierarchical levels in Figure 1 and Figure 2 in order to rectify and improve current design ideas. Such recognition can be facilitated through discussions with their peers, or answers to questions given by the educator. In Figure 13, I propose a hierarchical decision making tool that could facilitate the lateral and vertical transformations in design classrooms.

The advantages of the tool are multiple:

1. It is sufficiently open to be applied in all design related domains, and on all levels of expertise.

2. It is multi-layered nature allows for complexity of process, as well as knowledge acquisition and application.

3. Its multi-directionality allows for feedback loops that encourage reflection, critical and coherent thinking.

4. It is easy to use and adapt to a variety of contexts.

\section{Conclusion}

Designing, here, is considered as a process of activities related to decision making, physically and mentally, in a coherent way that is connected to the contextualised intentions of given design problems. Assisting design students in making decisions efficiently implies coherent, yet complex relational thinking. Instead of relying on restrictive knowledge-orientated, dual-nature thinking tools that focus on epistemology, an alternative decision making tool was proposed here. From the Theory of Decision Making, the ontology of hierarchical thinking, and the case study, it is apparent that decision making entails much more than thinking about artefacts and the functional norms that dictate the appropriate choice of physical elements. It is a personal process of incremental and non-linear connection making between aspectual intentions, functionbehaviour intentions, physical elements, and implementation intentions. It has been shown that this process is driven by designers anchoring their decisions in the various intentions, namely aspectual, functional and behavioural. Implementation intentions do not serve as an anchor, but are a further development of the concretisation and decision making process. Although it is distinguished as a separate level in the 4-level hierarchical model, thinking about implementation in intertwined with designers' thinking about physical elements. In this context, the proposed 4level hierarchical decision making tool has practical, educational, and theoretical value.

Design methodology could benefit from a tool that allows for more complex thinking processes. Against this background, the 4-level hierarchical decision making tool has educational as well as theoretical benefits. By virtue of its openness and multi-layered nature, the tool can assist educators in prompting students to think hierarchically yet multi-directionally. By providing multiple layers of thinking, emphasis is placed on the value of contextualised intentions, ensuring coherence and focus. The empirical evidence provided in the case study indicated that it is necessary to add aspectual implementations at the top of the hierarchy, which confirms the theory of Verkerk, Hoogland, van der Stoep and de Vries (2007). This theory finds that aspects form an important part of the characterisation of artefacts. The 4-level hierarchical decision making model furthermore provides a useful tool for researching the protocols of designers systematically when combined with the Theory of Lateral and Vertical Transformation. 
Using the tool for this purpose, educators are favourably positioned to apply a cognitive apprenticeship approach, which will guide their scaffolding processes. In turn, students can use the tool directly to assist in their problem solving process, and guide their decision making in a rational, yet non-linear manner. As a learning object, the 4-level hierarchical decision making tool can guide students' personalised problem solving processes individually, as well as in groups. It can serve as tool for internal idea generation. It may also encourage multiple forms of external representation, which could encourage internal as well as external conversations, judging, choosing, manipulating, developing, decision making and evaluating. Developing their representation skills, their sensitivity to relevant internal and external sources of information, and coherent connection making might therefore foster novices' design behaviour to mirror that of expert designers. The theoretical value of this paper lies within the empirical evidence of the cognitive complexity involved in four levels of thinking found in real design thinking. This extends current reductive theorising about the dual nature of artefacts and its application in design methodology. In addition, the complexity of the suggested tool and the evidence provided by the case study leaves room for further research. The following questions beg to be answered:

1. What cognitive role does the structural quality of designers' sketches play when judging, choosing, evaluating and committing to a decision?

2. What psychological principles underlie designers' ability to coherently draw relations between aspectual intentions and implementation intentions? and finally

3. What knowledge types can be associated with students' multi-directional transformation actions?

\section{References}

Anderson, M. L. (2003). Embodied Cognition: A field guide. Artificial Intelligence, 149(1), 91130

Anthony, W. S. (1973). Learning to discover rules by discovery. Journal of Educational Psychology, 64, 325-328.

Basden, A. (2000). The Aspectual Framework of Meaning. Retrieved from The Dooyeweerd Pages website: http://www.dooy.salford.ac.uk/contact.html

Brandstatter, V., Heimbeck, D., Malzacher, J. T. \& Frese, M. (2003). Goals Need Implementation Intentions: The Model of Action Phases Tested in the Applied Setting of Continuing Education. European Journal of Work and Organizational Psychology, 12(1), 37-59.

Buzan, T. (2005). Mind Map Handbook. London: Thorsons.

Cascetta, E. (2001). Transportation Systems Engineering: Theory and Methods. Dordrecht: Springer Science+Media.

Collins, A., Brown, J. S. \& Newman, S. E. (1987). Cognitive Apprenticeship: Teaching the Craft of Reading, Writing, and Mathematics. Illinois: University of Illinois at UrbanaChampaign.

Conlan, T. (2006). Formative assessment of classroom concept maps: the reasonable fallible analyser. Journal of Interactive Learning Research, 17(1), 15-36.

Cross, N. (2001). Design Cognition: Results from Protocol and Other Empirical Studies of Design Activity. In C. Eastman, M. McCracken \& W. Newstetter (Eds.), Design Knowing and Learning: Cognition in Design Education. Oxford: Elsevier. 
de Miranda, M. A. (2004). The Grounding of a Discipline: Cognition and Instruction in Technology Education. International Journal of Technology and Design Education, 14, 61-77.

De Vries, M., Custer, R. L., Dakers, J. R., \& Martin, G. (2007). Analyzing Best Practices in Technology Educatiion. Rotterdam: Sense Publishers.

de Vries, M. J. (2006). Technological Knowledge and Artifacts: An Analytical View. In J. R. Dakers (Ed.), Defining Technological Literacy. Towards an Epistemological Framework. New York: Pelgrave MacMillan.

Edelson, D. C., Gordin, D. N. \& Pea, R. D. (1999). Addressing the challenges of inquiry-based learning through technology and curriculum design. The Journal of the Learning Sciences, 8 (3\&4), 391-450.

Eder, W. E. (2012). Comparisons of several design theories and methods with the legacy of Vladimir Hubka.

Epley, N. \& Gilovich, T. (2006). The anchoring-and-adjustment heuristic. Why the adjustments are insufficient. Psychological Science, 17(4), 311-318.

Ertmer, P. A. \& Newby, T. J. (2013). Behaviorism, cognitivism, constructuvism: comparing critical feautres from an instructional design perspective. Performance Improvement Quarterly, 26(2), 43-71.

Fox, J., Cooper, R. P. \& Glasspool, D. W. (2013). A canonical theory of dynamic decisionmaking. Frontiers in Psychology, 4(150), 1-19.

Gavrilova, T., Leshcheva, I. \& Strakhovich, E. (2015). Gestalt principles of creating learning business ontologies for knowledge codification. Knowledge Management Research \& Practice, 13(4), 418-428.

Gero, J. S., \& Kannengieser, U. (2004). The situated function-behaviour-structure framework. Design Studies, 25, 373-391.

Gibson, J. J. (1986). The Ecological Approach to Perception. Hillside, NJ: Lawrence Erlbaum Associates.

Gigerenzer, G. \& Goldstein, D. G. (1996). Reasoning the fast and frugal way: Models of bounded rationality. Psychological Review, 103(4), 650-669.

Goel, V. (1995). Sketches of Thought. Cambridge: MIT Press.

Goldstein, W. M. \& Hogarth, R. M. (1997). Judgment and decision research: some historical context. In W. M. Goldstein \& R. M. Hogarth (Eds.), Research on Judgment and Decision Making: Currents, Connections and Controversies (pp. 3-68). Cambridge, UK: Cambridge University Press.

Gollwitzer, P. M. \& Schaal, B. (1998). Metacognition in Action: The Importance of Implementation Intentions. Personality and Social Psychology Review, 2(2), 124-136.

Hastie, R. (2001). Problems for judgment and decision making. Annual Review Psychology, 52, 653-683.

Haupt, G. (2013). The cognitive dynamics of socio-technological thinking in the early phases of expert designers' design process. (Unpublished PhD), University of Pretoria, Pretoria.

Haupt, G. (2015). Learning from experts: Fostering extended thinking in the early phases of the design process International Journal of Technology and Design Education, 25(4).

Hennessy, S. (1993). Situated Cognition and Cognitive Apprenticeship: Implications for Classroom Learning. Studies in Science Education, 22(1), 1-44.

Hofweber, T. (2014). Logic and Ontology. In E. N. Zalta (Ed.), The Stanford Encyclopedia of Philosophy (Vol. Fall 2014 Edition). Stanford: Standford University. 
Johnson, S. D. \& Daugherty, J. (2008). Quality and characteristics of recent research in technology education. Journal of Technology Education, 20(1), 16-31.

Jonassen, D. (1998). Designing constructivist learning environments. In C. M. Reigeluth (Ed.), Instructional Design Models and Strategies (2nd ed.). Mahwah NJ: Lawrence Erlbaum.

Katsikopoulos, K. V. (2009). Coherence and correspondence in engineering design: informing the converstation and connecting with judgment and decision-making research. Judgment and Decision Making, 4(2), 147-153.

Kirschner, P. A., Sweller, J. \& Clark, R. E. (2006). Why mininal guidance during instruction does not work: an analysis of the failure of constructivist, discover, problem-based, experiential, and inquir-based teaching. Educational Psychologist, 41(2), 75-86.

Kluge, P. \& Malan, D. F. (2011). The application of the analytical hierarchical process in complex mining engineering design problems. The Journal of the South African Institute of Mining and Metallurgy, 111(December), 847-855.

Kroes, P. A. (2002). Design Methodology and the Nature of Technical Artefacts. Design Studies(23), 287-302.

Kroes, P. A. \& Meijers, A. (2002). The Dual Nature of Technical Artifacts Techné, 6(2).

Lawson, B. (2006). How Designers Think. Boston: Elsevier.

Mitcham, C. (2002). Do Artifacts Have Dual Natures? Two Points of Commentary on the Delft Project. Techné, 6(2).

Mitcham, C. \& Holbrook, J. B. (2006). Understanding Technological Design. In J. S. Dakers (Ed.), Defining Technological Literacy. Towards an Epistemological Framework. New York: Palgrave MacMillan.

Oxman, R. (2002). The Thinking Eye: Visual Re-cognition in Design Emergence. Design Studies, 23(2), 135-164.

Oxman, R. (2004). Think-maps: teaching design thinking in design education. Design Studies, 25(1), 63-91.

Petrina, S. (2007). Advanced Teaching Methods for the Technology Classroom. London: Information Science Publishing.

Robbins, P. (2009). The Cambridge Handbook of Situated Cognition. Cambridge: Cambridge University Press.

Savin-Baden, M. (2007). Challenging PBL Models and Perspectives. In E. de Graaf \& A. Kolmos (Eds.), Management of Change: Implementation of Problem-Based and ProjectBased Learning in Engineering. Rotterdam: Sense Publishers.

Schön, D. (1984). Problems, frames and perspectives on designing. Design Studies, 5(3), 135156.

Seram, N. (2013). Decision making in product development - a review of the literature. International Journal of Engineering and Applied Sciences, 2(4), 1-11.

Simon, H. A. (1996). The Sciences of the Artificial (3rd ed.). Cambridge, MA: MIT Press.

Sowa, J. F. (1984). Conceptual Structures: Information Processing in Mind and Machine. Reading, MA: Addison-Wesley.

Suwa, M., Purcell, T. \& Gero, J. (1998). Macroscopic Analysis of Design Processes Based on a Scheme for Coding Designers' Cognitive Actions. Design Studies, 19(4), 455-483.

Suwa, M. \& Tversky, B. (1996). What Architects See in their Design Sketches: Implications for Design Tools. Paper presented at the Human Factors in Computing Systems, ACM, New York. 
Tversky, A. \& Kahneman, D. (1974). Judgement under uncertainty: heuristics and biases. Science, 185(4157), 1124-1131.

Tversky, A. \& Kahneman, D. (1986). Rational choice and the framing of decisions. The Journal of Business, 59(4), S251-S278.

Tversky, A. \& Simonson, I. (1993). Context-dependent preferences. Management Science, 39(10), 1179-1189.

Verkerk, M. J., Hoogland, J., van der Stoep, J. \& de Vries, M. J. (2007). Denken Ontwerpen Maken. Basisboek Techniekfolosofie. Amsterdam: Boom. 